\title{
Exoplanet Characterization by Proxy: A Transiting 2.15 R\# Planet Near the Habitable Zone of the Late K Dwarf Kepler-61
}

\section{Citation}

Ballard, Sarah, David Charbonneau, Francois Fressin, Guillermo Torres, Jonathan Irwin, JeanMichel Desert, Elisabeth Newton, et al. 2013. Exoplanet Characterization by Proxy: A Transiting 2.15 R\# Planet Near the Habitable Zone of the Late K Dwarf Kepler-61. The Astrophysical Journal 773, no. 2: 98. doi:10.1088/0004-637x/773/2/98

\section{Published Version}

doi:10.1088/0004-637X/773/2/98

\section{Permanent link}

http://nrs.harvard.edu/urn-3:HUL.InstRepos:29990215

\section{Terms of Use}

This article was downloaded from Harvard University's DASH repository, and is made available under the terms and conditions applicable to Other Posted Material, as set forth at http:// nrs.harvard.edu/urn-3:HUL.InstRepos:dash.current.terms-of-use\#LAA

\section{Share Your Story}

The Harvard community has made this article openly available.

Please share how this access benefits you. Submit a story.

Accessibility 


\title{
EXOPLANET CHARACTERIZATION BY PROXY: A TRANSITING $2.15 R_{\oplus}$ PLANET NEAR THE HABITABLE ZONE OF THE LATE K DWARF KEPLER-61
}

\author{
Sarah Ballard ${ }^{1,11}$, David Charbonneau ${ }^{1}$, Francois Fressin ${ }^{1}$, Guillermo Torres $^{1}$, Jonathan Irwin ${ }^{1}$, \\ Jean-Michel Desert ${ }^{2}$, Elisabeth Newton ${ }^{1}$, Andrew W. ManN ${ }^{3}$, David R. Ciardi ${ }^{4}$, Justin R. Crepp ${ }^{2,5}$, \\ Christopher E. Henze ${ }^{6}$, Stephen T. Bryson $^{6}$, Steven B. Howell ${ }^{6}$, Elliott P. Horch ${ }^{7}$, \\ MARK E. EveretT ${ }^{8}$, AND Avi SHPORER ${ }^{2,9,10}$ \\ ${ }^{1}$ University of Washington, Seattle, WA 98195, USA; sarahba@uw.edu \\ ${ }^{2}$ California Institute of Technology, Pasadena, CA 91125, USA \\ ${ }^{3}$ Institute for Astronomy, University of Hawai'i, Honolulu, HI 96822, USA \\ ${ }^{4}$ NASA Exoplanet Science Institute/Caltech, Pasadena, CA 91125, USA \\ ${ }^{5}$ Department of Physics, University of Notre Dame, Notre Dame, IN 46556, USA \\ ${ }^{6}$ NASA Ames Research Center, Moffett Field, CA 94035, USA \\ ${ }^{7}$ Southern Connecticut State University, New Haven, CT 06515, USA \\ ${ }^{8}$ National Optical Astronomy Observatory, Tucson, AZ 85719, USA \\ ${ }^{9}$ Las Cumbres Observatory Global Telescope Network, Santa Barbara, CA 93117, USA \\ ${ }^{10}$ Department of Physics, University of California, Santa Barbara, CA 93106, USA \\ Received 2012 September 5; accepted 2013 April 22; published 2013 July 30
}

\begin{abstract}
We present the validation and characterization of Kepler-61b: a $2.15 R_{\oplus}$ planet orbiting near the inner edge of the habitable zone of a low-mass star. Our characterization of the host star Kepler-61 is based upon a comparison with a set of spectroscopically similar stars with directly measured radii and temperatures. We apply a stellar prior drawn from the weighted mean of these properties, in tandem with the Kepler photometry, to infer a planetary radius for Kepler-61b of $2.15 \pm 0.13 R_{\oplus}$ and an equilibrium temperature of $273 \pm 13 \mathrm{~K}$ (given its period of $59.87756 \pm$ 0.00020 days and assuming a planetary albedo of 0.3 ). The technique of leveraging the physical properties of nearby "proxy" stars allows for an independent check on stellar characterization via the traditional measurements with stellar spectra and evolutionary models. In this case, such a check had implications for the putative habitability of Kepler-61b: the planet is $10 \%$ warmer and larger than inferred from $K$-band spectral characterization. From the Kepler photometry, we estimate a stellar rotation period of 36 days, which implies a stellar age of $>1 \mathrm{Gyr}$. We summarize the evidence for the planetary nature of the Kepler-61 transit signal, which we conclude is 30,000 times more likely to be due to a planet than a blend scenario. Finally, we discuss possible compositions for Kepler-61b with a comparison to theoretical models as well as to known exoplanets with similar radii and dynamically measured masses.
\end{abstract}

Key words: eclipses - planetary systems - stars: individual (Kepler-61, KOI 1361, KIC 6960913)

Online-only material: color figures

\section{INTRODUCTION}

With the discoveries of exoplanets Kepler-22b (Borucki et al. 2011), Kepler-20e and f (Fressin et al. 2012), Kepler-42a, b, and c (Muirhead et al. 2012a), Kepler-68c (Gilliland et al. 2013), and Kepler-62e and f (Borucki et al. 2013), astronomers are encroaching upon the regime of transiting terrestrial exoplanets in their stellar habitable zones. Kepler-22b is the first superEarth-sized exoplanet with a measured radius to reside in the habitable zone of a Sun-like star, though its radius of $2.4 R_{\oplus}$ does not necessitate a terrestrial composition. The Kepler-20, Kepler-42, and Kepler-68 exoplanetary systems each comprise multiple planets, some of which are Earth-sized or smaller (as small as Mars in the case of Kepler-42c). However, these planets orbit too close to their host stars to lie within the habitable zone. The star Kepler-62 hosts five planets, two of which are both very likely solid and reside in their star's habitable zone (Borucki et al. 2013). The most recent release of Kepler exoplanetary candidates (Batalha et al. 2013) contains 10 members $<2 R_{\oplus}$ and with equilibrium temperatures between 185 and $303 \mathrm{~K}$. This temperature range is a generous definition of the habitable zone

\footnotetext{
${ }^{11}$ NASA Carl Sagan Fellow.
}

proposed by Kasting (2011). Half of these candidate exoplanets orbit stars cooler than $4100 \mathrm{~K}$, as reported by the Kepler Input Catalog (KIC).

However, inferring the properties of low-mass stars from spectra (upon which a measurement of planetary radius and equilibrium temperature hinges so critically) presents difficulties on multiple fronts. The direct comparison of theoretical spectra to observations, which is robust for deducing the properties of solar-type stars, is challenging for low-mass stars. Such spectra rely on detailed, computationally intensive modeling of convection in low-mass stellar interiors (Mullan \& MacDonald 2001; Browning 2008) and complete lists of the complex array of molecules and grains that reside in their atmospheres (Tsuji et al. 1996; Allard et al. 2000). For this reason, we often appeal to empirical, rather than theoretical, methods for the physical characterization of low-mass stars (see Torres 2013 for a complete review). This challenge is compounded by the possibility that stellar properties may also depend on other parameters, such as activity and metallicity, in a significant way. The empirical technique for deriving $\mathrm{M}$ dwarf temperatures and metallicities from $K$-band spectra that was innovated by Rojas-Ayala et al. (2012) offers an important inroad. However, this technique depends upon the $\mathrm{H}_{2} \mathrm{O}-\mathrm{K} 2$ spectral index, which is an effective probe 
of stellar temperature for mid-M dwarfs, but saturates for stars with temperatures higher than $3900 \mathrm{~K}$ (Muirhead et al. 2012b). There exists a desert in stellar temperature, near $4000 \mathrm{~K}$, where no reliable method for temperature derivation from a spectrum exists: the $\mathrm{H}_{2} \mathrm{O}-\mathrm{K} 2$ index has saturated, and the star is yet too cool for comparison between high-resolution optical spectra and synthetic models. This is an especially salient problem for the characterization of the current and future sample of exoplanets orbiting low-mass stars, given the astonishing occurrence rates of 1.0 planet/star (found from the Kepler sample to be $0.90_{-0.03}^{+0.04}$ planets/star per Dressing \& Charbonneau 2013 and $1.0 \pm 0.1$ planets/star per Swift et al. 2013).

There exists an alternative means of measuring the properties of the nearest and brightest low-mass stars, with measured distances from parallax: interferometric measurements of their radii. The radius measurement, in tandem with the bolometric flux, also enables a direct measurement of the stellar temperature with minimal modeling uncertainties. The number of low-mass stars with directly measured properties from interferometry is growing, and this pool of stars can be plumbed for proxies to stars too faint for such direct characterization themselves. Muirhead et al. (2012a) undertook the first such analysis with an application of the properties of Barnard's star toward a characterization of the M4V star and three transiting planets comprising the Kepler-42 system. A similar method would be especially useful for a star astride the $4000 \mathrm{~K}$ boundary, where spectroscopic estimates of the stellar properties may be unreliable, and for an exoplanetary host star. Kepler-61 is such a star: its temperature lies near $4000 \mathrm{~K}$, and, depending upon the source of its stellar characterization, the equilibrium temperature of its planet lies either outside or astride the stellar habitable zone. The radius of the planet varies from 2.0 to $2.3 R_{\oplus}$ (Muirhead et al. 2012a and NASA Exoplanet Archive, respectively $)^{12}$ depending on the assumed size of the star, which range brackets both a plausible rocky composition (more amenable to habitability) or a "mini-Neptune" composition. Moreover, the now exists a sample of four stars with (1) similar spectral type and (2) direct radius and temperature measurements, which can be applied to break the degeneracy of the planet's putative habitability.

The apparent magnitude of Kepler-61, with Kepler magnitude $\mathrm{Kp}=15.0$, renders the star too dim to enable a radial velocity measurement of the planet's mass. However, even without a mass measurement of Kepler-61b, we are able to validate its authentic planetary nature with a statistical argument about the likelihood of the planet scenario in comparison to false-positive scenarios. We undertake such an analysis with BLENDER, which machinery has already been applied to validate Kepler-9d (Torres et al. 2013), Kepler-11g (Lissauer et al. 2011), Kepler-10c (Fressin et al. 2011), Kepler-19b (Ballard et al. 2011), Kepler-22b (Borucki et al. 2012), and Kepler-20 e and $\mathrm{f}$ (Fressin et al. 2012). In this case, a single observation of the transit depth at $4.5 \mu \mathrm{m}$ with Warm Spitzer in tandem with the BLENDER result, plays a prominent role in ruling out hierarchical triple false-positive scenarios.

In Section 2, we describe the Kepler observations of Kepler-61. In Section 3, we describe our characterization of the transit light curve and the physical parameters of the star, including our method of applying the properties of nearby similar stars to characterize Kepler-61. In Section 4, we describe the

\footnotetext{
12 http://exoplanetarchive.ipac.caltech.edu/cgi-bin/ExoTables/nph-exotbls?
} dataset=cumulative validation of Kepler-61b as an authentic planet with BLENDER. We include a description of follow-up imaging observations of the star to characterize any additional stars within the Kepler photometric aperture of Kepler-61, as measurements of the photocentroid gather from the Kepler images and a measurement of the transit depth with Warm Spitzer. In Section 5, we first explore plausible compositions for the planet, given the growing number of transiting super-Earth-sized planets with dynamically measured masses. We close this section with a discussion of future prospects.

\section{KEPLER OBSERVATIONS}

The Kepler spacecraft, launched on 2009 March 7, is photometrically monitoring 170,000 stars for $8 \mathrm{yr}$ for evidence of transiting planets. Argabright et al. (2008) provides an overview of the Kepler instrument, and Caldwell et al. (2010) and Jenkins et al. (2010b) provide a summary of its performance since its launch. The Kepler observations of Kepler-61 (KIC number 6960913) that we present in this work were gathered from 2009 May 13 to 2012 October 3, spanning Kepler "Quarters" 1-14. All data for this star were gathered in long-cadence mode (characterized by an exposure time of 29.5 minutes) for Quarters $1-11$, and in short-cadence mode (characterized by an exposure time of 58.5 s) for Quarters 12-14. The data contain gaps of approximately three days between quarters for scheduled downlinks. Kepler-61b was first identified as exoplanetary candidate Kepler Object of Interest (KOI) 1361.01 by Borucki et al. (2011). We employed the light curves generated by the Kepler aperture photometry (PDC-Map) pipeline, described in Twicken et al. (2010), to which we add an additional step. We remove the effects of baseline drift by individually normalizing each transit as follows. We fit a linear function of time to the flux immediately before and after transit (specifically, from $9 \mathrm{hr}$ to 20 minutes before first contact, equal to 2.5 transit durations, and an equivalent time after fourth contact).

\section{ANALYSIS}

\subsection{Derivation of Stellar Parameters}

While the physical characterization of isolated low-mass stars is a notoriously difficult problem (Ségransan et al. 2003; Torres 2013), several recent techniques have offered promising inroads by tying spectra of $\mathrm{M}$ dwarfs to directly measured quantities. Such characterization of low-mass stars is crucial, as the Kepler mission has demonstrated that low-mass stars are hosts to small planets at a rate of 1.0 planet/star (Dressing \& Charbonneau 2013; Swift et al. 2013).

\subsubsection{Characterization in the Literature}

Kepler-61 is classified as an M0 star by Muirhead et al. (2012b), who employed $K$-band spectra of the star to infer stellar properties (in this work, they refer to Kepler-61b by its KOI notation, KOI 1361.01). They measure ratios of equivalent widths of $\mathrm{Na}$ and $\mathrm{Ca}$ to determine the stellar metallicity, and deformation between continuum regions within $K$-band (the $\mathrm{H}_{2} \mathrm{O}-\mathrm{K} 2$ index, first developed by Covey et al. 2007 and recalibrated by Rojas-Ayala et al. 2012), which they interpolate onto the theoretical metallicity and $\mathrm{H}_{2} \mathrm{O}-\mathrm{K} 2$ surface from the BT-Settl late-type model spectra (Allard et al. 2012) to determine the stellar effective temperature. The metallicity relation published by Rojas-Ayala et al. (2012) is calibrated using binaries comprising an M dwarf and an FGK star, which 


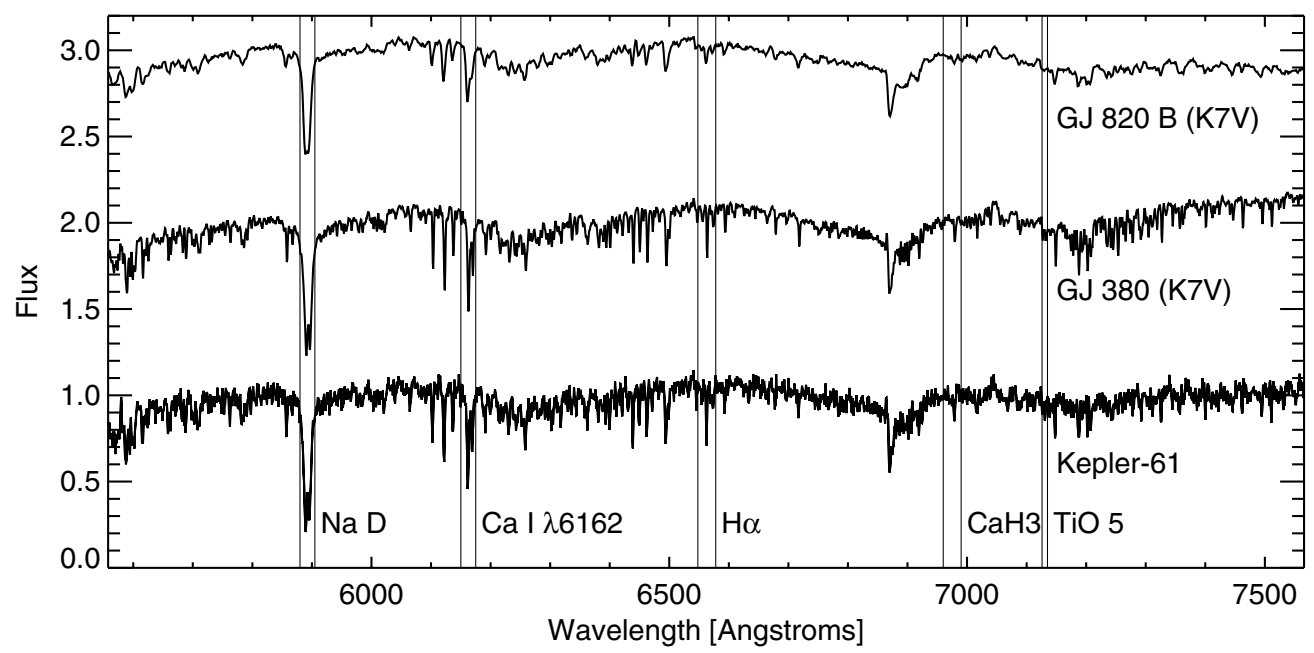

Figure 1. FAST spectra of GJ 820B (top), GJ 380 (center), and Kepler-61 (bottom). We have denoted the wavelength regions employed by spectral typing software, "The Hammer," developed by Covey et al. (2007).

Muirhead et al. (2012b) then applied to a set of isolated lowmass stars in the Kepler sample. For Kepler-61, they find $T_{\text {eff }}$ of $3929_{-135}^{+66} \mathrm{~K}$ and metallicity $[\mathrm{Fe} / \mathrm{H}]$ of $-0.02 \pm 0.11$ (though they caution that applying the $K$-band metallicity metric to stars with temperatures higher than $3900 \mathrm{~K}$ relies upon an extrapolation of the Rojas-Ayala et al. 2012 metric). By comparing this temperature and metallicity to the Dartmouth stellar evolutionary models (Dotter et al. 2008), they determine a stellar radius $R_{\star}=0.55 \pm 0.07 R_{\odot}$ and a mass $M_{\star}=$ $0.57 \pm 0.07 R_{\odot}$. However, as described above, this location in temperature space is close to the location where the $\mathrm{H}_{2} \mathrm{O}-\mathrm{K} 2$ index saturates (for $T_{\text {eff }}>3900 \mathrm{~K}$; Muirhead et al. 2012b), and the deformation between continuum regions is too small to effectively probe stellar temperature. Dressing \& Charbonneau (2013) corroborates the result that temperatures derived from the $\mathrm{H}_{2} \mathrm{O}-\mathrm{K} 2$ index are significantly lower than those derived from the comparison of the broadband colors to models for stars near the $3900 \mathrm{~K}$ marker. The Dressing \& Charbonneau (2013) method relies upon a comparison of the measured magnitudes of the star from the KIC (2MASS $J H K$ and Sloan filters $g, r$, $i$, and $z$ ) against the colors predicted from the Dartmouth stellar evolutionary models (Dotter et al. 2008). They assign a prior on stellar metallicity based upon the metallicity distribution of the $\mathrm{M}$ dwarfs observed in the Casagrande et al. (2008) sample and a prior on height above the galactic midplane similar to that applied by Brown et al. (2001) for the KIC. For the colors of Kepler-61, they estimate an effective temperature of $4060_{-109}^{+100 K} \mathrm{~K}$, a radius of $0.57_{-0.11}^{+0.06} R_{\odot}$, and a mass of $0.57_{-0.09}^{+0.08} M_{\odot}$ (C. Dressing 2013, private communication).

\subsubsection{Optical and Near-infrared Spectroscopy}

Using the FAST spectrograph on the $1.5 \mathrm{~m}$ telescope at Mount Hopkins, AZ, we gathered a spectrum of Kepler-61 in the range 5560-7570 $\AA$ with $0.75 \AA$ resolution (we employed an integration time of 20 minutes to achieve a signal-to-noise ratio of 30). In Figure 1, we show the Kepler-61 spectrum in comparison to spectra of two nearby K7V stars, GJ 380 and GJ 820B, gathered with the same instrument (we observed the former on 2012 April 24 with integration time $10 \mathrm{~s}$ for a signal-to-noise ratio of $210 \mathrm{pixel}^{-1}$ and gathered the latter spectrum from the FAST Spectrograph Archive. It was observed on 2010 November 4 at resolution of $1.5 \AA$ and an integration time of $2 \mathrm{~s}$ for a signal-to-noise ratio of 200 pixel $^{-1}$ ). We have denoted the wavelength regions employed by spectral typing software, "The Hammer," developed by Covey et al. (2007), which we use to determine a spectral type of K7V for the KOI. Lépine et al. (2013) find that classification with the Hammer agrees with stellar classification from spectral indices within 1.0 subtypes.

We consider the set of similarly typed stars with directly measured radii. While spectral types for stars in the M0-K7 range compiled in the literature often vary by one to two subtypes, we defer to the spectral types listed in Boyajian et al. (2012). In this case, because the Dressing \& Charbonneau (2013) predicted temperature for Kepler-61 encompasses an effective temperature as high as $4160 \mathrm{~K}$ (within $1 \sigma$ ), we elect to exclude K5 stars from the sample of spectroscopically similar stars with resolved radii: the K5 stars GJ 820A and GJ 720B have effective temperatures of $4361 \pm 17$ (Kervella \& Fouqué 2008; van Belle \& von Braun 2009; Boyajian et al. 2012) and $4393 \pm$ 149 (Boyajian et al. 2012) respectively; their lowest temperature estimates within $1 \sigma$ are still hotter than the $1 \sigma$ range predicted for temperature of Kepler-61 from broadband photometry. The sample of K7 and M0 stars with radius measurements currently comprises four stars: GJ 380 (radius and temperatures measured gathered by Lane et al. 2001; van Belle \& von Braun 2009, and Boyajian et al. 2012), GJ 338A (measurements from Boyajian et al. 2012), GJ 338B (measurements from Boyajian et al. 2012), and GJ 820B (measurements from Kervella \& Fouqué 2008 and van Belle \& von Braun 2009). We list the properties of these stars in Table 1.

We have compiled the set of $K$-band spectra for these stars in addition to the KOI, which we depict in Figure 2, in order to estimate their $[\mathrm{Fe} / \mathrm{H}]$ metallicities in a uniform fashion from the metric of Mann et al. (2013; which metric is valid for stars as early as spectral type K5). Our spectrum for Kepler-61 was gathered and published by Muirhead et al. (2012a) with the TripleSpec instrument at Palomar Observatory (Herter et al. 2008). They employed an exposure time of 6 minutes on 2011 June 6, with resolution of $3 \AA$ ( $R$ of 2700$)$, to obtain a signalto-noise ratio of $60 \mathrm{pixel}^{-1}$. We gathered spectra for GJ 338A and GJ 338B on 2013 January 27 with the SpeX instrument at NASA's Infrared Telescope Facility (IRTF; Rayner et al. 2003), and for GJ 380 on 2012 December 17. We employed the ShortXD observing mode (resolution of $5 \AA$, $R$ of 2000) 

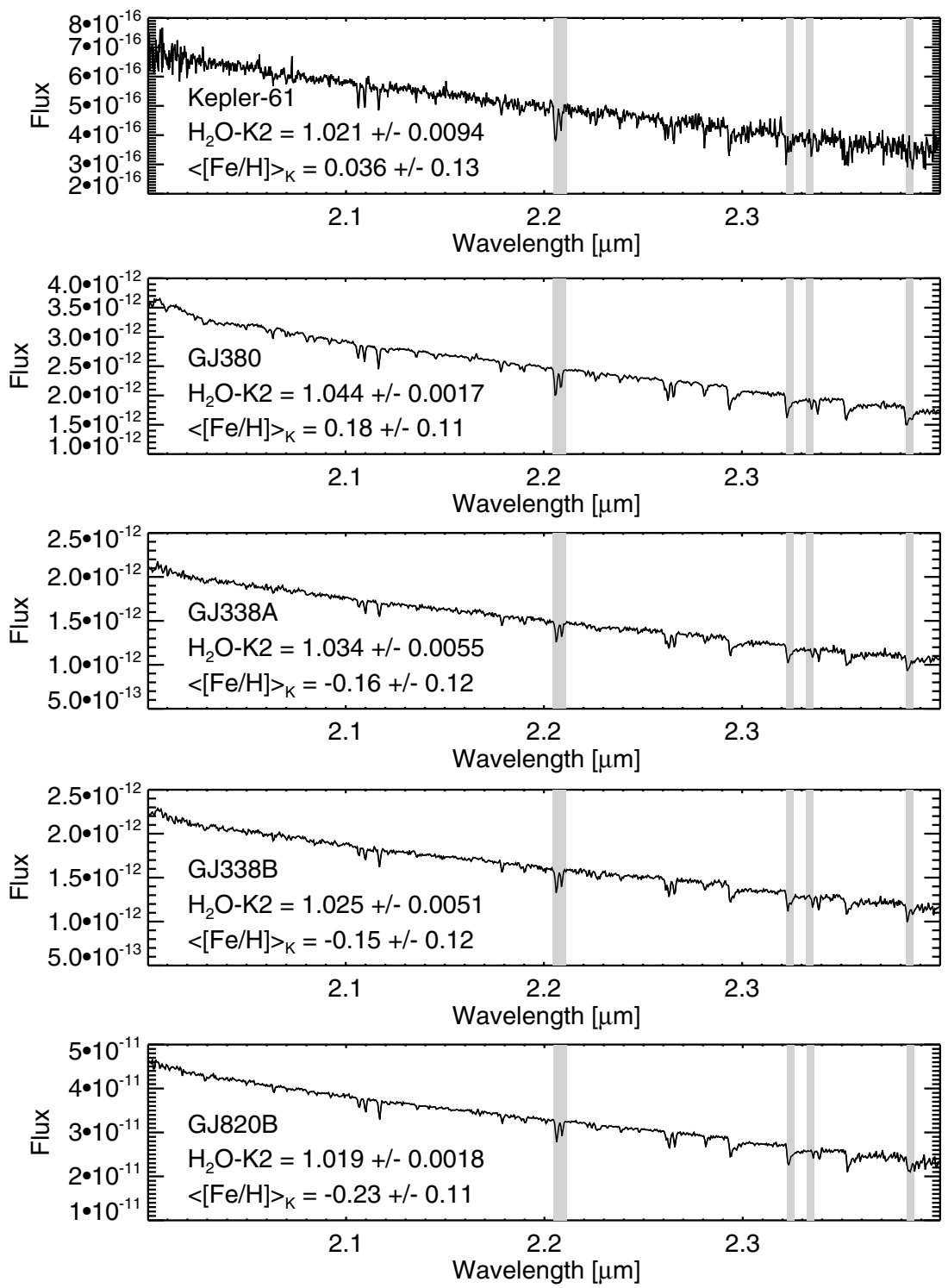

Figure 2. $K$-band spectra for Kepler-61 (KOI 1361) and for nearby stars of similar spectral type. Overplotted in grey are the metal-sensitive regions published by (Mann et al. 2013); we use the metric described in that work to calculate $[\mathrm{Fe} / \mathrm{H}]$ for each star. The $\mathrm{H}_{2} \mathrm{O}-\mathrm{K} 2$ temperature index (Rojas-Ayala et al. 2012) is also stated for each star.

Table 1

Comparison of Observables between Kepler-61 and Similar Stars

\begin{tabular}{lccccc}
\hline \hline Parameter & GJ 380 & GJ 338A & GJ 338B & GJ 820B & Kepler-61 \\
\hline Spectral type & K7V & M0V & K7V & K7V & K7V \\
Metallicity [Fe/H] & $0.18 \pm 0.11$ & $-0.15 \pm 0.12$ & $-0.15 \pm 0.12$ & $-0.23 \pm 0.11$ & $0.03 \pm 0.14$ \\
$\mathrm{H}_{2} \mathrm{O}-\mathrm{K} 2$ & $1.044 \pm 0.002$ & $1.034 \pm 0.005$ & $1.025 \pm 0.005$ & $1.019 \pm 0.002$ & $1.02 \pm 0.010$ \\
$E_{\mathrm{H} \alpha}{ }^{\mathrm{b}}$ (in absorption) & -0.61 & -0.56 & -0.50 & -0.59 & -0.82 \\
$\log \left(L_{X} / L_{\text {bol }}\right)^{\mathrm{a}}$ & -5.16 & -4.68 & -4.65 & -5.03 & $\cdots$ \\
Rotation period (days) $^{\mathrm{c}}$ & 11.67 & $\cdots$ & 10.17 & $37.9 / 48$ & 36 \\
${\text { Estimate age }(\mathrm{Gyr})^{\mathrm{c}}}^{\mathrm{C}}$ & 0.2 & 0.1 & $\cdots$ & 3 & $\cdots$ \\
\hline$R_{\star}\left(R_{\odot}\right)^{\mathrm{a}}$ & $0.64 \pm 0.004$ & $0.58 \pm 0.01$ & $0.57 \pm 0.01$ & $0.60 \pm 0.007$ & $0.62_{-0.05}^{+0.02}$ (adopted) \\
$T_{\text {eff }}(\mathrm{K})^{\mathrm{a}}$ & $4085 \pm 14$ & $3907 \pm 35$ & $3867 \pm 35$ & $3932 \pm 25$ & $4017_{-150}^{+6}($ adopted) \\
\hline
\end{tabular}

Notes.

${ }^{a}$ Values for standard stars from Boyajian et al. (2012).

${ }^{\mathrm{b}}$ Values for standard stars from Gizis et al. (2002).

${ }^{\mathrm{c}}$ From Barnes (2007), stated to nearest $100 \mathrm{Myr}$. 

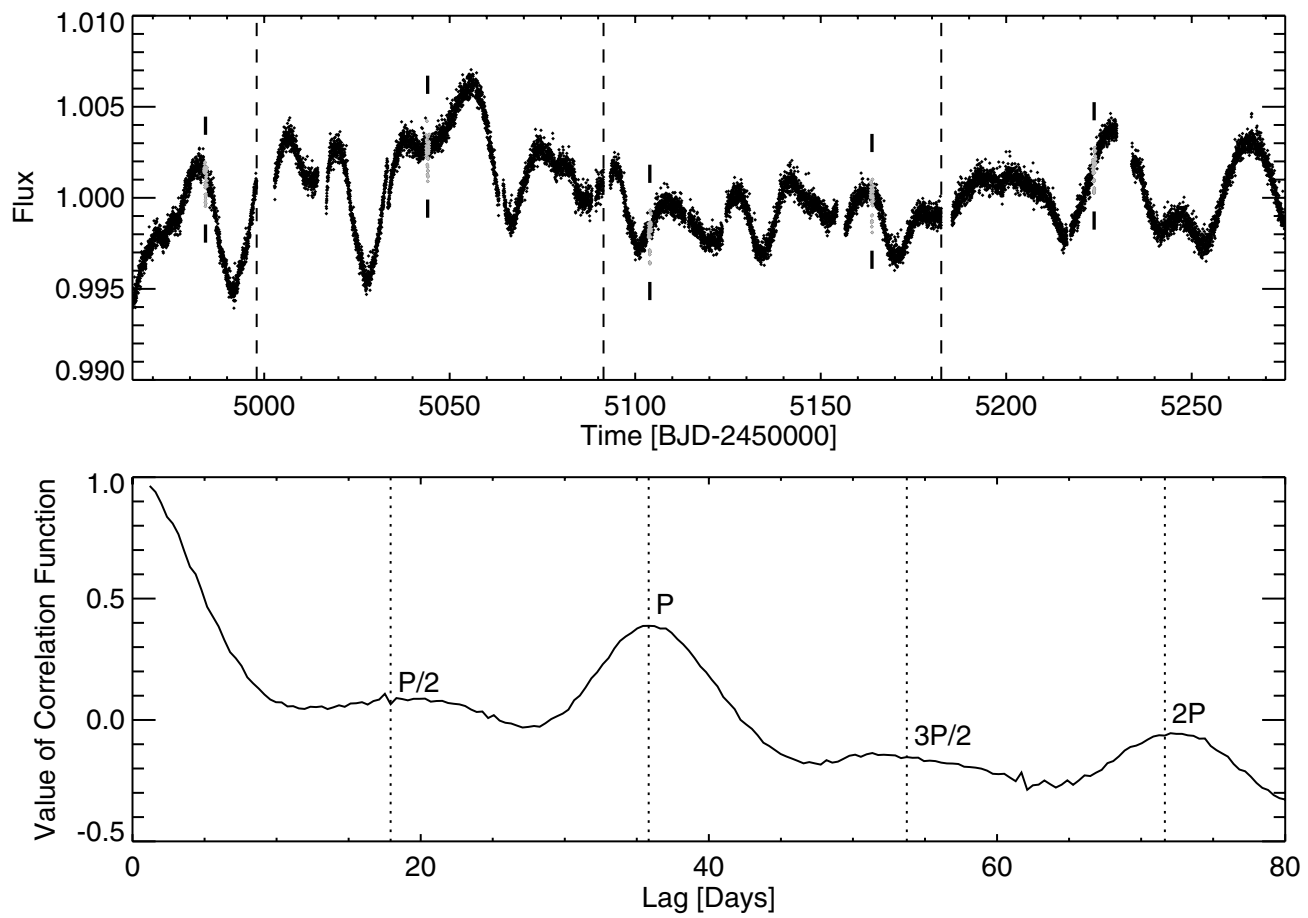

Figure 3. Top: Quarters 1-4 of Kepler observations of Kepler-61. Dashed lines denote the intervals between quarters. We indicate the times of transit of Kepler-61 in grey, with vertical lines immediately above and below the transit observations. Bottom: the results of the discrete correlation function applied to this portion of the light curve. The strongest peak corresponds to 36 days, which variability is present by eye in the 300 days of observations depicted in the top panel. Dotted lines indicate the strongest period and its harmonics.

and exposure times of $1 \mathrm{~s}, 1 \mathrm{~s}$, and $5 \mathrm{~s}$, respectively, for signalto-noise ratios of 150,150 , and 700 pixel $^{-1}$. We gathered our spectrum of GJ 820B from the IRTF Spectral Library (Rayner et al. 2009). It was observed on 2001 October 20 with the same resolution, and has a signal-to-noise ratio of 800 pixel $^{-1}$. We compute uncertainties on these metallicities from adding the scatter in the $K$-band metric quoted by Mann et al. (2013) of 0.11 dex in quadrature to the intrinsic uncertainty in the value of $[\mathrm{Fe} / \mathrm{H}]_{K}$ from the error in the spectrum at the wavelengths that are operative for the metric. We list these derived metallicities in Table 1, but note that no standard star possesses a metallicity consistent with $1 \sigma$ of the most probable $[\mathrm{Fe} / \mathrm{H}]$ value for Kepler-61 of 0.03. Unlike in the case of Kepler-42 (Muirhead et al. 2012b), none of these nearby stars possesses features consistent enough with the KOI to render one of them an single ideal "proxy" star for the Kepler target.

\subsubsection{Activity and Age Indicators}

We consider the activity levels of the stars in this sample, in comparison to Kepler-61. We have measured the equivalent widths in $\mathrm{H} \alpha$ for both Kepler-61 from the FAST spectra to those compiled by Gizis et al. (2002). They exhibit equivalent widths in $\mathrm{H} \alpha$ (all in absorption) ranging from -0.82 (for Kepler-61) to -0.50 (for GJ 338A). In addition, we have measured the rotation period for Kepler-61 from the Kepler photometry, which we compare to the rotation periods listed for three of the four nearby stars in Barnes (2007). The "gyrochronology" technique of mapping the rotational period of a star to its age is described in detail in Barnes (2003, 2007), and is used specifically in Barnes (2007) to estimate ages of 1.96 Myr, 1.36 Myr, and 2.96 Gyr for GJ 380, GJ 338A, and GJ 820, respectively. In Figure 3, we depict the first four quarters of Kepler observations of Kepler-61, or approximately $1 \mathrm{yr}$ of continuous observation. These observations have been processed using the Presearch Data Conditioning (PDC) module of the Kepler data analysis pipeline, with the Bayesian Maximum A Posteriori (MAP) approach applied (described in Smith et al. 2012). The use of highly correlated and quiet stars to create a set of co-trending basis vectors enables the removal of non-astrophysical artifacts from the Kepler time series, and the preservation of astrophysically interesting signals such as stellar rotation. We apply the discrete correlation function of Edelson \& Krolik (1988), similarly applied by Fabrycky et al. (2012) on the time series of Kepler-30 and Queloz et al. (2009) on CoRoT-7, on this portion of the Kepler-61 light curve. We test lags from 1 to 100 days, and identify a $36 \pm 4$ day periodicity, which we attribute to the stellar rotation period. In Figure 3, we show both the Kepler photometry and autocorrelation function that we employed to characterize the stellar rotation. Irwin et al. (2011) recently published a compilation of the known rotation periods of low-mass stars from the literature. The rotation periods are drawn from open clusters of stars with derived ages from 1-650 Myr (which ages are measured by main sequence fitting of these clusters, as compared to stellar evolutionary models), and then also from field stars with ages $>1$ Gyr. Among the clusters with ages less than $1 \mathrm{Gyr}$, nearly all of the stars with masses $>0.5 M_{\odot}$ have rotation periods shorter than 30 days, and only after $1 \mathrm{Gyr}$ do low-mass stars with masses greater than $0.5 M_{\odot}$ appear to spin down enough to produce 36 day rotation periods. Stars with masses between 0.5 and $0.7 M_{\odot}$ and ages between 8 and 10 Gyr are more likely to have rotation periods in the tens of days (Kiraga \& Stepien 2007 and Baliunas et al. 1996 observed values $<30$ days for field stars in the 1-2 Gyr range). We therefore take the observed rotation period of Kepler- 61 to be conservatively indicative of an age $>1$ Gyr.

We also apply the age metric of Barnes (2010) to estimate the age of Kepler-61, which Swift et al. (2013) used to obtain an 


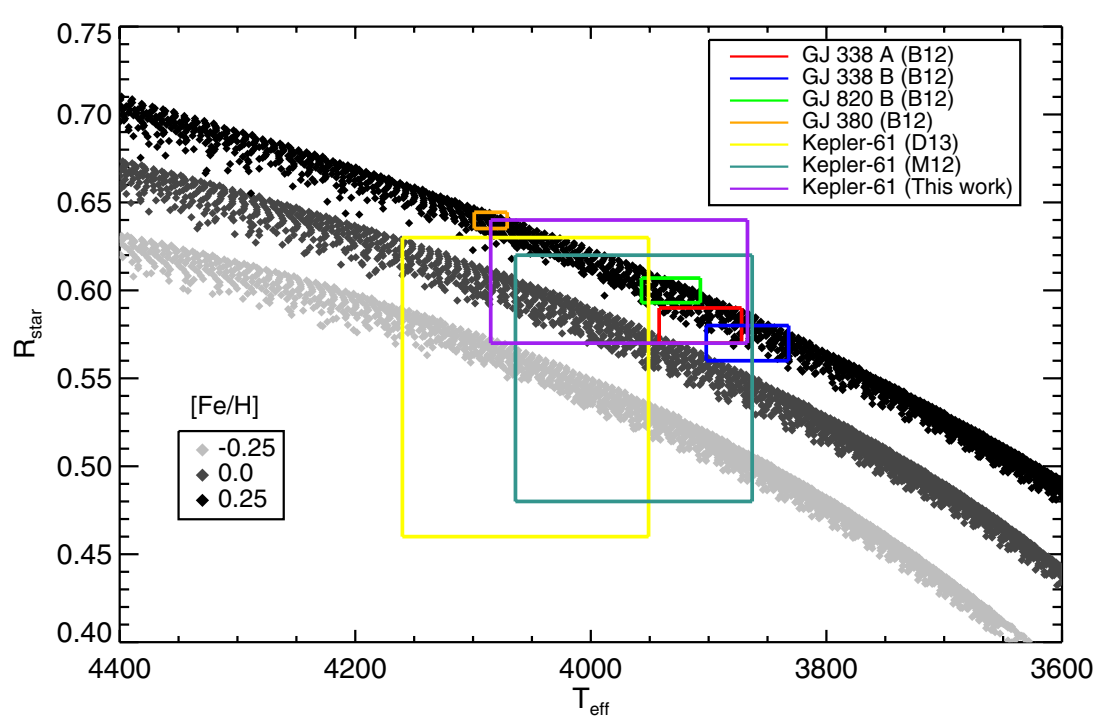

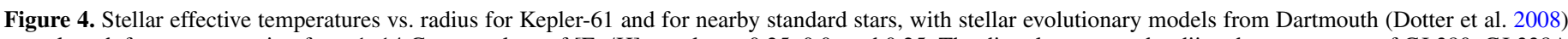

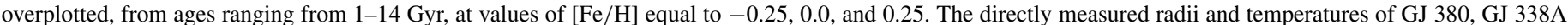

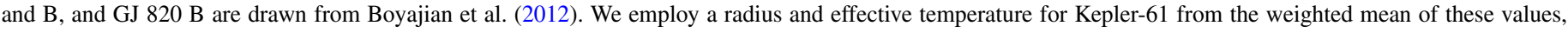

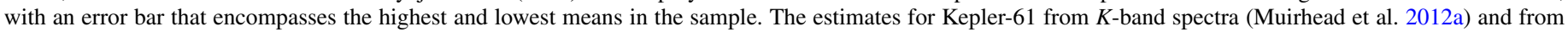
the broadband KIC photometry (Dressing \& Charbonneau 2013) are also depicted.

(A color version of this figure is available in the online journal.)

age approximation for Kepler-32:

$$
t=\frac{\tau}{k_{C}} \ln \left(\frac{P}{P_{0}}\right)+\frac{k_{I}}{2 \tau}\left(P^{2}-P_{0}^{2}\right),
$$

where the dimensionless constants $k_{C}=0.646$ days $\mathrm{Myr}^{-1}$ and $k_{I}=452 \mathrm{Myr} \mathrm{day}^{-1}$ are approximated in Barnes (2010). We estimate the convective turnover time $\tau$ from Wright et al. (2011), who derived empirical convective turnover times for a sample of 824 solar and late-type stars, the typical convective turnover time of a star within the mass range $0.47-0.62 M_{\odot}$ is 29 days. It is not possible to determine the initial spin period $P_{0}$ associated with Kepler-61 at its birth, so we defer to the median value of the initial spin period, $P_{0}=2.81$ days required to produce the observed rotation rates for $0.6 M_{\odot}$ stars in the Praesepe cluster (Agüeros et al. 2011) similarly to Swift et al. (2013). This formulation returns an age for Kepler-61 of $10 \mathrm{Gyr}$. We therefore find that ages between 1-10 Gyr are consistent with different metrics of age constraints for Kepler-61, and simply adopt a lower bound on its age of $1 \mathrm{Gyr}$.

Because none of these nearby stars comprises an ideal "proxy" to the planet-host star (i.e., possessing both statistically indistinguishable metallicities and temperature indices in tandem with similar activity indicators), we adopt the conservative tack of employing a radius and temperature for Kepler-61 which are the weighted mean of the radii and temperatures of the set of standard stars. For our uncertainty on these values, we encompass the highest and lowest mean value among the sample. We therefore adopt for Kepler-61 a radius of $0.62_{-0.05}^{+0.02} R_{\odot}$ and a temperature of $4017_{-150}^{+68} \mathrm{~K}$. In Figure 4, we show this $1 \sigma$ confidence interval for the radius and temperature of Keper-61 in comparison to the values of four nearby standard stars from Boyajian et al. (2012) and also in comparison to the values for Kepler-61 published by Dressing \& Charbonneau (2013) and by Muirhead et al. (2012a).

\subsection{Derivation of Planetary Parameters}

We estimated the uncertainty in the planetary transit parameters using the Markov Chain Monte Carlo (MCMC) method as follows. We employ model light curves generated with the routines in Mandel \& Agol (2002), which depend upon the period $P$, the epoch $T_{c}$, the planet-to-star radius ratio $R_{p} / R_{\star}$, the ratio of the semi-major axis to the stellar radius $a / R_{\star}$, the impact parameter $b$, the eccentricity $e$, and the longitude of periastron, $\omega$. We fixed two quadratic limb-darkening coefficients (LDCs), $u_{1}$ and $u_{2}$, to theoretical values based on the adopted effective temperature and radius. We employed the theoretical LDCs generated for the Kepler bandpass by Claret \& Bloemen (2011) from the PHOENIX models corresponding to a star with $T_{\text {eff }}$ of $4000 \mathrm{~K}$ and solar metallicity, which is the closest match to the effective stellar temperature $T_{\text {eff }}$ of 4017 and the metallicity of $0.03 \pm 0.14$ for Kepler-61 (we additionally specified a $\log (g)$ of 4.5 and an intermediate turbulent velocity value of $2 \mathrm{~km} \mathrm{~s}^{-1}$ ): these coefficients are $u_{1}=0.50, u_{2}=0.20$. We accounted for the 29.5 minute integration time of the Kepler photometry, which is 3 times longer than the ingress and egress duration of the planet candidate, by evaluating the light curve model at intervals of 1 minute, and then summing the model over the long cadence integration time. We model the three quarters of short cadence observations independently (gathered from Quarters 12-14, during which time the planet presented five transits). For these short cadence observations, we evaluate the light curve model at each time measurement, in $58.5 \mathrm{~s}$ intervals, and fix the period and transit time to the best-value recovered over the 11 quarter baseline of long-cadence observations.

To generate the MCMC chain, we randomly choose one parameter, perturb it, and evaluate the $\chi^{2}$ of the solution. If the $\chi^{2}$ is lower, we accept the new parameter value. If the $\chi^{2}$ is higher, we evaluate the probability of accepting the jump as $p=e^{-\Delta \chi^{2} / 2}$. If the jump is rejected, the procedure is repeated at that point in the chain until an acceptable jump occurs. We adjust the width of the distribution from which we randomly draw the jump sizes in each parameter until $20 \%-40 \%$ of jumps 
are executed in each of the parameters. We created five chains, each of length $10^{6}$ points, where each of the chains is begun from a different set of starting parameters (each parameter is assigned a starting position that is $+3 \sigma$ or $-3 \sigma$ from the bestfit values). We discard the first $20 \%$ of jumps from each chain to remove the transient dependence of the chain on the starting parameters. We first conducted this analysis, as described, using only the Kepler light curve to inform our value of $\chi^{2}$. However, the allowable stellar densities we infer from the light curve alone are much broader than the range of stellar densities consistent with our spectroscopic information about the star. Because Kepler-61 is a late $\mathrm{K}$ star, it is slowly evolving-therefore, its range of theoretical densities is tightly constrained for ages $<14$ Gyr. When we apply the MCMC algorithm toward fitting the long-cadence transit parameters independently and allow $a / R_{\star}$ to float, we find that values of $a / R_{\star}$ from $35-150$ furnish comparable fits to the light curve (the ingress and egress time, at $9.3 \pm 3.2$ minutes measured at long-cadence, results in a wide family of allowable light-curve fits). We take advantage of two circumstances that allow us to better constrain the transit parameters. First, there exist five transits gathered by Kepler at short-cadence, where the ingress and egress time are resolved by the one-minute exposure time. Second, we make use of the fact that low-mass stars are slowly evolving to set a physically motivated prior on $a / R_{\star}$ as follows. We based our procedure for constraining the mass, radius, and age of the host star on the method described by Torres et al. (2008). Using the metallicity we derive from $K$ band, we created a set of stellar evolution models from the Dartmouth isochrone series (Dotter et al. 2008). We employed the interpolation software that accompanied that work, which accepts as inputs the age of the star, the iron abundance, and the abundance of $\alpha$-elements relative to solar (for which we assume the solar value), and outputs a grid of stellar isochrones corresponding to a range of masses. We evaluated a set of isochrones over an age range of $1-14 \mathrm{Gyr}$ (at intervals of $0.1 \mathrm{Gyr}$ ) and in $[\mathrm{Fe} / \mathrm{H}]$ in increments of 0.01 from $-0.5-0.5$ dex (encompassing $3 \sigma$ above and below the measured $[\mathrm{Fe} / \mathrm{H}]$ of $0.03 \pm 0.14)$. We evaluate the physical radius corresponding to each stellar model via $\log (g)$ and the mass of the $\operatorname{star}\left(g=G M_{\star} / R_{\star}^{2}\right)$.

Rearranging Kepler's version of Newton's third law in the manner employed by Seager \& Mallén-Ornelas (2003), Sozzetti et al. (2007) and Torres et al. (2008), we convert the period (derived from photometry), and the radius and mass of the host star (from isochrones) to a ratio of the semi-major axis to the radius of the host star, $a / R_{\star}$ :

$$
\frac{a}{R_{\star}}=\left(\frac{G}{4 \pi^{2}}\right)^{1 / 3} \frac{P^{2 / 3}}{R_{\star}}\left(M_{\star}+M_{p}\right)^{1 / 3},
$$

where we will hereafter assume that $M_{p}$ is negligible when compared to the mass of the host star.

We calculate the corresponding value of $a / R_{\star}$ for each stellar model. We then generate the MCMC chain as follows. We implement a prior on $a / R_{\star}$ by varying the adopted quantities $R_{\star}$ and $T_{\text {eff }}$ in the chain, in addition to the light curve parameters. For each set of $R_{\star}$ and $T_{\text {eff }}$, we locate the closest stellar model associated with these values and record its corresponding stellar density, $a / R_{\star}$. It is this value of $a / R_{\star}$ that is used to generate the light curve model, along with the other light curve parameters $P, T_{c}, R_{p} / R_{\star}, b, e$, and $\omega$ (defined above), which are permitted to vary independently. We assign uniform flat priors in $b$ (from $0-1$ ), $e$ (from $0-1$ ), and $\omega$ (from $0-2 \pi$ ), and uniform improper priors on all other parameters. In this way, we
Table 2

Star and Planet Parameters for Kepler-61

\begin{tabular}{|c|c|}
\hline Parameter & Value and $1 \sigma$ Confidence Interval \\
\hline \multicolumn{2}{|c|}{ Kepler-61 (star) } \\
\hline Right ascension ${ }^{\mathrm{a}}$ & $19^{\mathrm{h}} 41^{\mathrm{m}} 13^{\mathrm{s}} .09$ \\
\hline Declination $^{\mathrm{a}}$ & $+42^{\mathrm{d}} 28^{\mathrm{m}} 31^{\mathrm{s}} .0$ \\
\hline$T_{\text {eff }}(\mathrm{K})^{\mathrm{b}}$ & $4017_{-150}^{+68}$ \\
\hline$R_{\star}(\text { Solar radii })^{\mathrm{b}}$ & $0.62_{-0.05}^{+0.02}$ \\
\hline$M_{\star}($ Solar masses $)$ & $0.635 \pm 0.037$ \\
\hline$[\mathrm{Fe} / \mathrm{H}]^{\mathrm{c}}$ & $0.03 \pm 0.14$ \\
\hline Age (Gyr) & $>1$ \\
\hline \multicolumn{2}{|c|}{ Kepler-61 (planet) } \\
\hline Period (days) & $59.87756 \pm 0.00020$ \\
\hline$T_{0}(\mathrm{BJD}-2,450,000)$ & $4984.1880_{-0.0024}^{+0.0029}$ \\
\hline$R_{p} / R_{\star}$ & $0.03301 \pm 0.00085$ \\
\hline $\mathrm{a} / R_{\star}$ & $90.6 \pm 3.4$ \\
\hline inc (deg) & $>89.80$ \\
\hline$e$ & $<0.25$ \\
\hline$e \cos (\omega)$ & $0.0 \pm 0.29$ \\
\hline Impact parameter & $<0.29$ \\
\hline Total duration (min) & $290.7 \pm 4.6$ \\
\hline Ingress duration (min) & $9.56 \pm 0.47$ \\
\hline$R_{p}($ Earth radii $)$ & $2.15 \pm 0.13$ \\
\hline Planetary $T_{\mathrm{eq}}(\mathrm{K})$ & $273 \pm 13$ \\
\hline
\end{tabular}

Notes.

a ICRS (J2000) coordinates from the 2MASS All-Sky Catalog of Point Sources (Cutri et al. 2003). The proper motion derived by Roeser et al. (2010) is

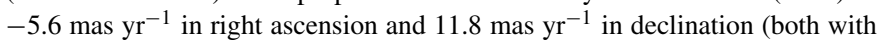

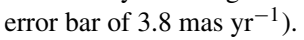

b Stellar temperature and radius inferred from weighted mean of directly measured K7 and M0 sample, as described in text.

${ }^{\text {c }}$ Metallicity derived from $K$-band using metric from Mann et al. (2013).

are sampling only values of $a / R_{\star}$ that are consistent with the spectroscopically derived parameters, but values of $a / R_{\star}$ that are not as well matched to the light curve are penalized by the $\chi^{2}$ term corresponding to the photometry. We adopt Gaussian priors on $R_{\star}$ and $T_{\text {eff }}$, which we implement by adding extra terms in the $\chi^{2}$ (where $\mathbf{P}$ corresponds to the vector of light curve parameters at each iteration):

$$
\chi^{2}=\sum_{i=1}^{n}\left(\frac{f_{i}-m(\mathbf{P})_{i}}{\sigma_{i}}\right)^{2}+\left(\frac{\Delta T_{\mathrm{eff}}}{\sigma_{T_{\mathrm{eff}}}}\right)^{2}+\left(\frac{\Delta R_{\star}}{\sigma_{R_{\star}}}\right)^{2} .
$$

In Figure 5, we show the correlations between the posterior distributions of subset of parameters in the model fit, as well as the histograms corresponding to each parameter. In Figure 6, we show the phased Kepler transit light curve for Kepler-61b, with the best-fit transit light curve overplotted. We report the best-fit parameters and uncertainties in Table 2. The range of acceptable solutions for each of the light curve parameters is determined as follows. Following Torres et al. (2008), we report the most likely value from the mode of the posterior distribution, marginalizing over all other parameters. The uncertainty is derived from the extent of the posterior distribution that encloses $68 \%$ of values closest to the mode.

\subsection{Physical Parameters}

This procedure described in Section 3.2 is also advantageous in that, in addition to recording the value of $a / R_{\star}$ at each iteration of the MCMC chain, we may also record the other traits of the 


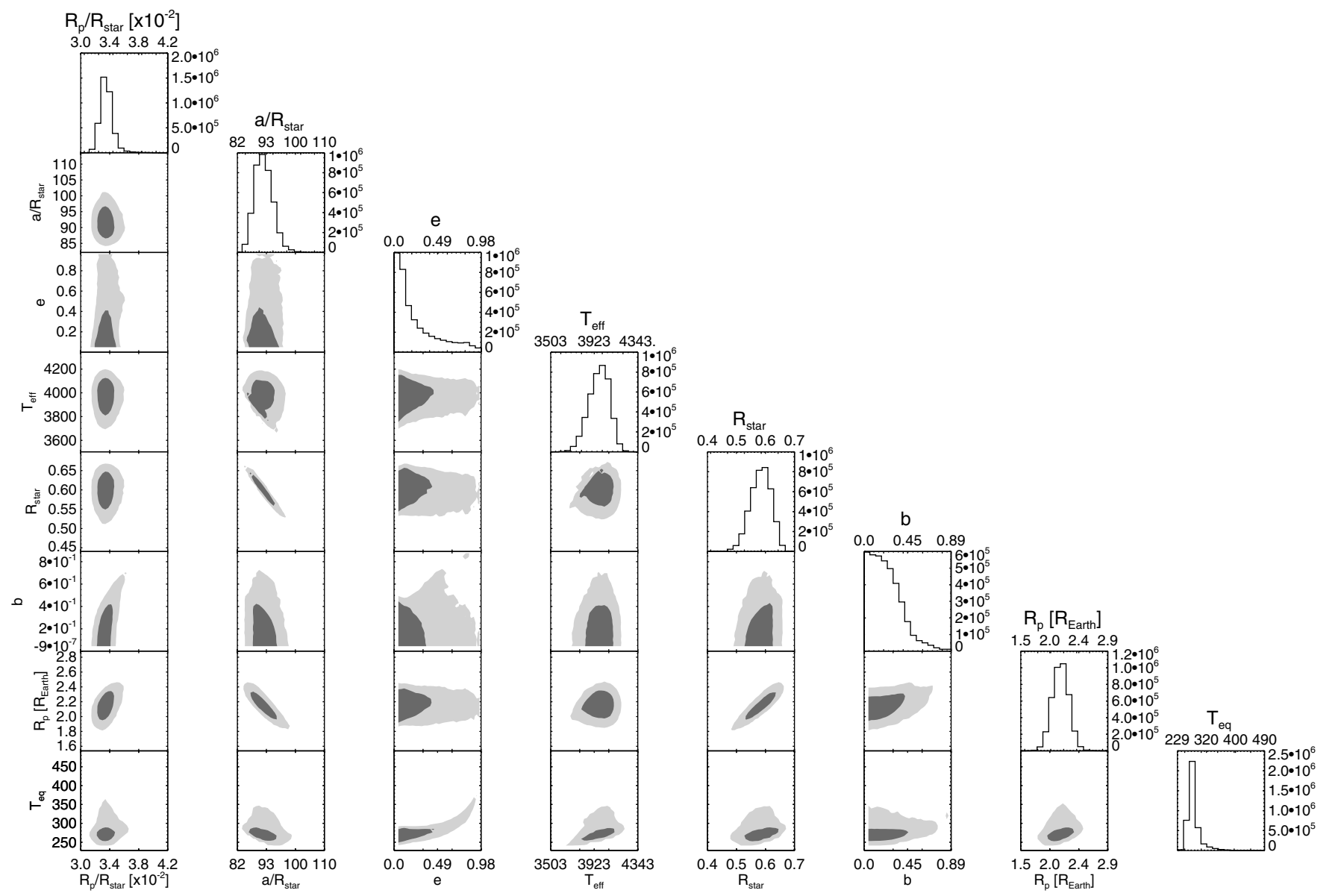

Figure 5. Markov Chain Monte Carlo probability distributions for light curve parameters of Kepler-61. The dark gray area encloses $68 \%$ of the values in the chain, while the light gray area encloses $95 \%$ of the values. We assign the range of values corresponding to $1 \sigma$ confidence from the area enclosing $68 \%$ of the values nearest to the mode of the posterior distribution for each parameter (as described in the text).
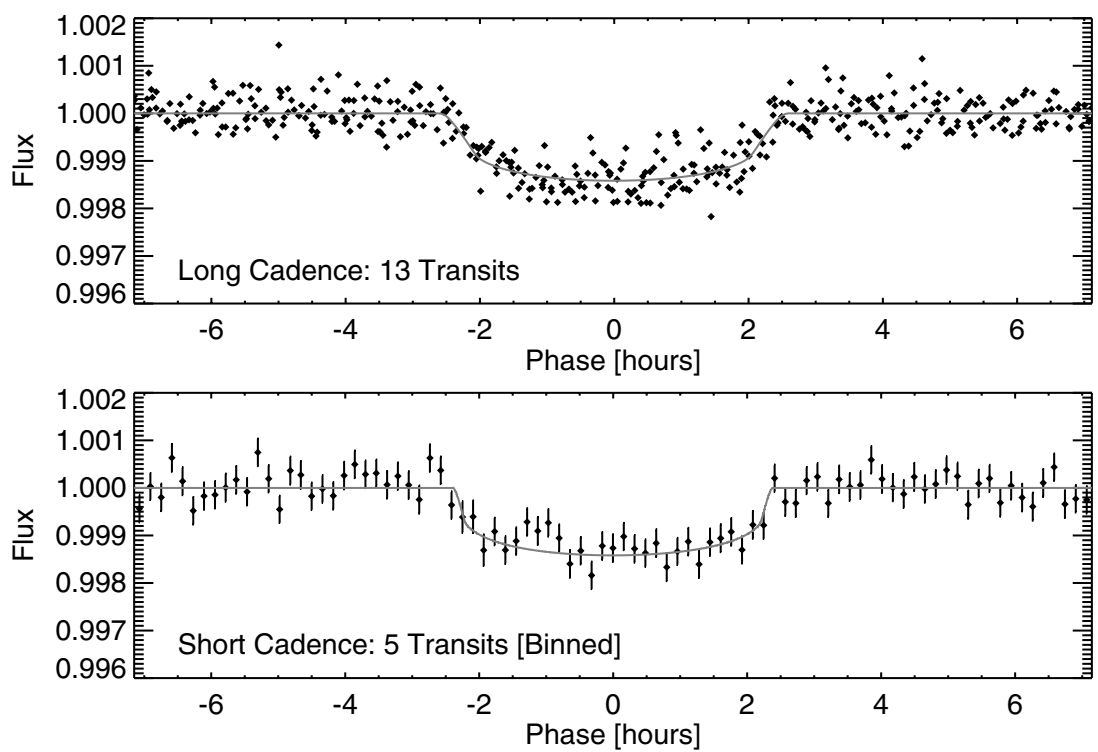

Figure 6. Top panel: Kepler-61 transit light curve as a function of phase for Quarters 1-11, gathered in long-cadence observing mode. Bottom panel: Kepler light curve for Quarters 12-14, gathered in short-cadence observing mode and binned in intervals of 7 minutes. Overplotted in grey is the best transit model light curve, with parameters given in Table 2 . The effect of integrating over the 29.5 minute exposure time corresponding to long-cadence observations is apparent in the different shapes of transit during ingress and egress. 

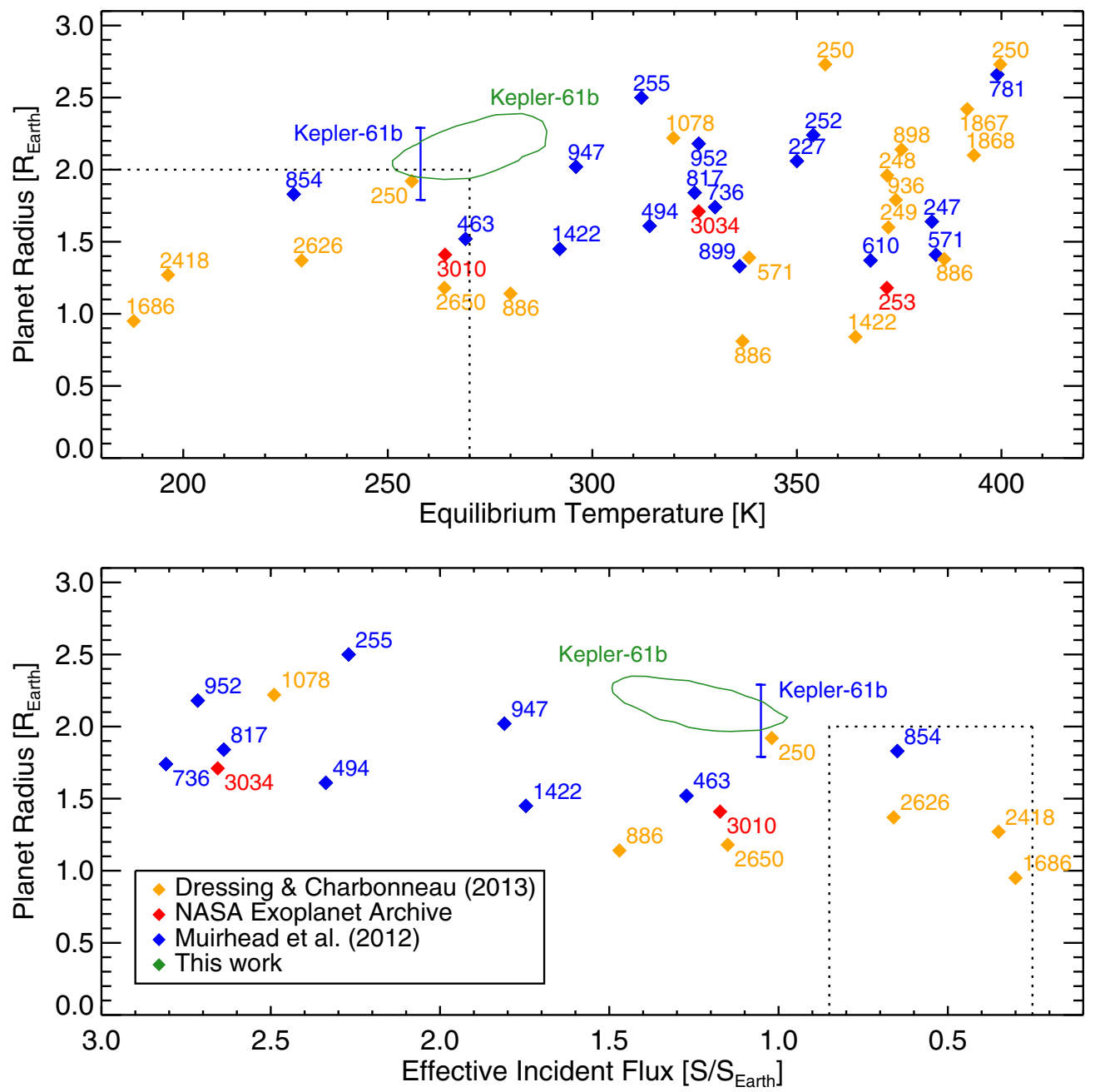

Figure 7. Top panel: sample of Kepler candidate exoplanets with radii $<3 R_{\oplus}$, orbiting stars with $T_{\text {eff }}<4200 \mathrm{~K}$, and with equilibrium temperatures $<400 \mathrm{~K}$. The dotted lines denote $270 \mathrm{~K}$ (above which runaway greenhouse effect occurs, per Kaltenegger \& Sasselov 2011), and $2 R_{\oplus}$. Bottom panel: sample of Kepler candidate exoplanets with radii $<3 R_{\oplus}$, orbiting stars with $T_{\text {eff }}<4200 \mathrm{~K}$, and incident flux levels within three times the value received at the surface of the Earth. The dotted lines here denote the habitable zone for stars from $2700-4500$ K, per Kopparapu et al. (2013). The number labels adjacent to points depict the KOI number. The values for planet candidates calculated by Muirhead et al. (2012b) orbiting stars with $T_{\text {eff }}<3800 \mathrm{~K}$ are depicted in blue. For candidates orbiting hotter stars, for which the $K$-band method tends to underpredict temperature, we depict values instead from the NASA Exoplanet Archive in red (this stellar characterization relies instead upon the comparison of the broadband colors to stellar models of Pinsonneault et al. 2012). Where these latter values have been revised by Dressing \& Charbonneau (2013) using the Dartmouth stellar models (Dotter et al. 2008), we have used those radius and temperature values and depicted the KOI in gold. The radius and temperature for Kepler-61 (KOI 1361) from $K$-band spectroscopy reported by Muirhead et al. (2012b) is given by the blue error bar, while the revised $1 \sigma$ contour from this work is shown in green.

(A color version of this figure is available in the online journal.)

star at that value of $R_{\star}$ and $T_{\text {eff }}$, including its mass, luminosity, and age. At the conclusion of the MCMC analysis, therefore, we have accumulated a chain not only for the light curve parameters, but for the physical parameters as well, as predicted from evolutionary models. The correlations between parameters, both physical parameters and those associated with the light curve, are therefore preserved in the chain and incorporated into our estimate of the stellar parameters (although we note that we have not accounted for possible correlated error between our adopted values of effective temperature and radius measurements of the star). We calculate the planetary radius from multiplying the elements of the $R_{p} / R_{\star}$ by the chain of $R_{\star}$. We infer a value for the stellar mass from its posterior distribution of $M_{\star}=0.635 \pm$ 0.037. The slowly evolving nature of Kepler-61 results in a largely unconstrained estimate of stellar age. The stellar rotation period (36 days, described in Section 3.1.3) indicates a star older than $1 \mathrm{Gyr}$, which is consistent, though also itself only a weak constraint. It is also possible to evaluate the posterior distribution of planetary equilibrium temperatures from the MCMC analysis. In the case of a circular orbit, we require only the stellar radius and temperature, the planetary semimajor axis, and the planetary albedo. However, in the case of an eccentric orbit, the planet receives time-variable stellar insolation. In order to evaluate the equilibrium temperature of the planet in the case of nonzero eccentricity, we evaluate the time-averaged equilibrium temperature by performing an integral over the mean anomaly from 0 to $2 \pi$, using the formalism detailed in Murray \& Correia (2010), where $A$ is the geometric albedo of the planet, $d$ is its distance from the star, and $M$ is the mean anomaly:

$$
\frac{1}{2 \pi} \int_{0}^{2 \pi} T_{\mathrm{eq}} d M=\frac{1}{2 \pi}(1-A)^{1 / 4} \int_{0}^{2 \pi} \sqrt{\frac{R_{\star}}{2 d}} T_{\star} d M .
$$

In Figure 7, we show the $1 \sigma$ confidence interval for planetary radius and time-averaged temperature for Kepler-16, in comparison to the values for the ensemble of KOIs orbiting 

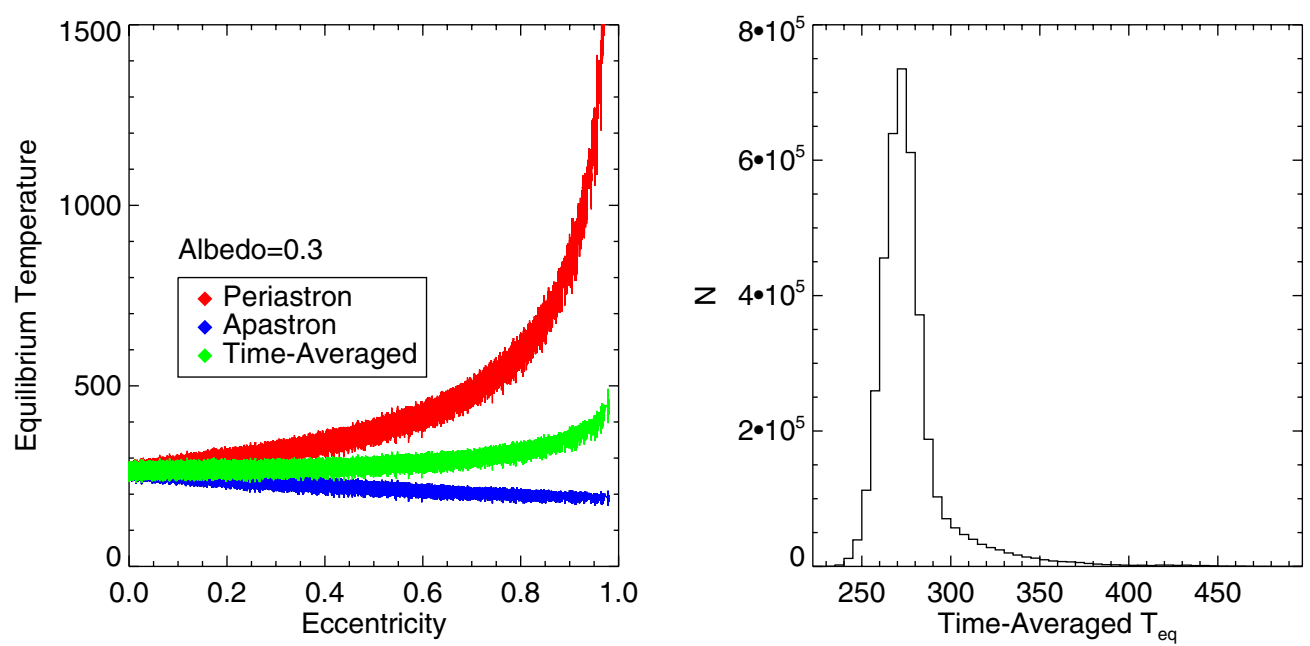

Figure 8. Left: for each iteration of the MCMC chain, we calculate the temperature of the planet at apastron (blue), periastron (red) and the time-averaged equilibrium temperature (green). We have assumed an albedo of 0.3. Right: histogram of time-averaged equilibrium temperatures for Kepler-61b. The long tail of temperatures higher than $350 \mathrm{~K}$ is contributed entirely from orbital eccentricities $>0.8$.

(A color version of this figure is available in the online journal.)

low-mass stars reported by Muirhead et al. (2012a), Dressing \& Charbonneau (2013), and NASA Exoplanet Archive. We also depict the stellar isolation versus planetary radius of these objects. Isolation was employed by Kopparapu et al. (2013) to assess habitability, in lieu of equilibrium temperature. In Figure 8, we show for Kepler-61 the planetary temperatures based on the apastron and periastron isolation from the star, as well as the time-averaged temperature of the planet, for each element of the MCMC chain.

We imposed a flat prior on the eccentricity of Kepler-61 from $0-1$, so the posterior distribution on $e$ includes values as high as 0.9 (such a large eccentricity still matches the transit duration at finely tuned values of $\omega$ ). Indeed, the circularization timescale for Kepler-61 has not yet elapsed if we assume it did not possess a large initial eccentricity. We consider the expression for circularization time (for modest initial $e$ ) given by Goldreich $\&$ Soter (1966), where $a$ is the semimajor axis of the planet, $R_{p}$ is the planetary radius, $M_{p}$ is the planetary mass, $M_{\star}$ the stellar mass, $Q$ is the tidal quality factor for the planet (which is highly uncertain, but we test two values: 100 for the assumption of a terrestrial composition, per Goldreich \& Soter 1966 , or $10^{4}$, which is the lower limit measured by Banfield \& Murray 1992 for Neptune) and $G$ is the gravitational constant:

$$
t_{\mathrm{circ}}=\frac{4}{63} \frac{1}{\sqrt{G M_{\star}^{3}}} \frac{M_{p} a^{13 / 2} Q}{R_{p}^{5}} .
$$

We find that the circularization timescale would be $400 \mathrm{Myr}$ for a terrestrial $Q$ of 100 (assuming a $7 M_{\oplus}$ planet orbiting a $0.64 M_{\odot}$ star at $0.25 \mathrm{AU}$ ), and $190 \mathrm{Gyr}$ for a Neptunelike $Q$ of $10^{4}$. It is therefore plausible that the planet resides in an eccentric orbit. If Kepler-61 began with a large initial eccentricity, then terms of the order of $\left(1-e^{2}\right)$ become relevant and the circularization timescale decreases (as elucidated in Equations (7)-(9) of Socrates et al. 2012). It is therefore also possible that the circularization timescale has indeed elapsed, dependent upon the initial eccentricity and tidal $Q$ of the planet. However, the uncertainty of our knowledge about its initial eccentricity (coupled with the uncertainty about the correct value of tidal $Q$ for Kepler-61 in particular) is such that we believed a flat prior on $e$ to be appropriate.
We find consistent orbital parameters for the 11 quarters of long-cadence observations (13 transits) and the 3 quarters of short cadence observations ( 5 transits), though our ability to resolve the shape of ingress and egress with short-cadence mode, coupled with the prior on $a / R_{\star}$ from our knowledge of the stellar parameters, is reflected in the error bars on $a / R_{\star}$ and the ingress/egress time $\tau$, even though we have only half the number of transits in the latter mode. While we find $\tau=9.3 \pm$ 3.2 minutes from the 13 transits in long-cadence mode, we find $\tau=9.56 \pm 0.47$ minutes from the 5 transits in short-cadence mode.

\section{PLANETARY VALIDATION OF KEPLER-61}

Morton \& Johnson (2011) provide a priori false positive probabilities for the Kepler planetary candidates published by Borucki et al. (2011), within which sample Kepler-61 is included. They cite the vetting of candidates by the Kepler software (detailed by Batalha et al. 2010) as being already sufficient to produce a robust list of candidates, and combine stellar population synthesis and galactic structure models to demonstrate that nearly all of these 1235 candidates have a false positive probability $<10 \%$. Kepler-61b, with a Kepler magnitude of 14.995 and a galactic latitude of 9.6 , has an a priori false positive probability of $4.8 \%$.

\subsection{Adaptive Optics Imaging}

We place limits on the presence of additional stars in the neighborhood of Kepler-61 with adaptive optics (AO) observations gathered at Keck with the NIRC2 instrument. On 2012 June 22 we observed Kepler-61 in both $J$ and $K$ band, with observed FWHM of the core in $K$ of 0.06 and in $J$ of $0^{\prime \prime} .10$. We detect a companion star 2.9 mag fainter and located 0.94 to the northwest of Kepler-61, which is shown in Figure 9. The high resolution $K$-band AO image cleanly resolves these two sources, while we employed the $J$-band image to better characterize the neighboring source. The additional source falls within the Kepler aperture, but is removed enough from the target star that we can employ the Kepler centroids to assert that the planet orbits the brighter star, as we describe in Section 4.3. The blended magnitudes are 15.0 in the Kepler bandpass, 

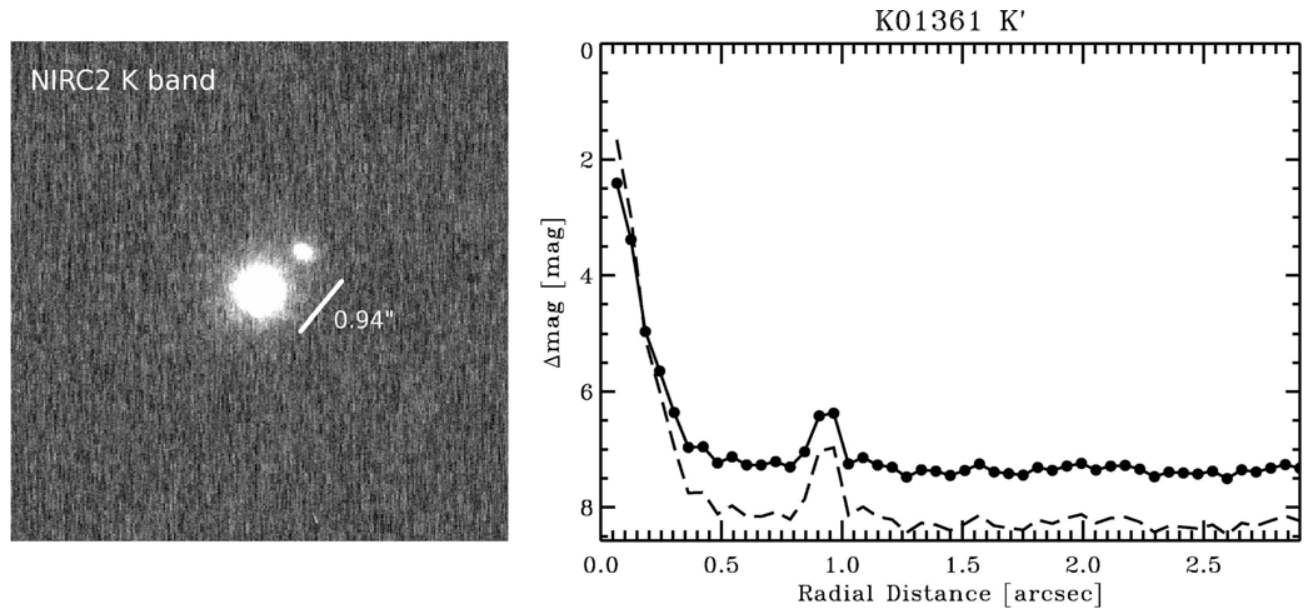

Figure 9. Left: $K$ band adaptive optics image of Kepler-61. An additional companion is located 0.94 away from the target star, and is 2.9 mag fainter. Right: the sensitivity limits to additional point sources in the neighborhood of Kepler-61 as a function of radial distance from the primary target. The filled circles represent the $K$ magnitude limits and each point represents a step in FWHM away from the primary target centroid peak. The dashed line underneath represents the $K$-band limits converted to Kepler magnitude limits if a star were to have a nominal $\mathrm{Kp}-K$ color, as described in the text.

$J=13.077 \pm 0.022 \mathrm{mag}$ and $K=12.272 \pm 0.019 \mathrm{mag} . \mathrm{We}$ independently measure the $J$ and $K$ magnitudes for the two stars, and apply the $\mathrm{Kp}-K$ and $J-K$ relationships (which are derived separately for dwarfs and giants from the KIC; this conversion is described in detail in Appendix A of Howell et al. 2012) to determine the de-blended Kepler magnitude. For the primary target, we find $\mathrm{Kp}=15.22 \pm 0.09 \mathrm{mag}, J=13.149 \pm$ $0.022 \mathrm{mag}$, and $K=12.345 \pm 0.019 \mathrm{mag}$. For the secondary, we find $\mathrm{Kp}=18.20 \pm 0.10 \mathrm{mag}, J=16.064 \pm 0.025 \mathrm{mag}$, and $K=15.242 \pm 0.020 \mathrm{mag}$. The two stars exhibit indistinguishable $J-K$ colors, with $0.804 \pm 0.029$ for Kepler-61 and $0.822 \pm 0.32$ for the dimmer companion.

We assess our sensitivity to additional sources using a similar procedure to that described by Batalha et al. (2011). We inject fake sources near the target star at random position angles, using steps in magnitude of $0.5 \mathrm{mag}$ and varying the distance from the target star in increments of 1.0 FWHM of the point-spread function. We then attempt to identify the injected sources with the DAOPhot routine (Stetson 1987) and also by eye and set our sensitivity limit as a function of distance at the magnitude where we are able to recover the injected sources. The limit in $\Delta m$ as a function of distance from the target star is shown in Figure 9. We then convert the $\Delta m$ sensitivity limit in $K$ band to a limit in Kepler magnitudes, again using the $\mathrm{Kp}-K$ relationship detailed in Howell et al. (2012).

\subsection{Speckle Imaging}

We gathered speckle imaging of Kepler-61 on 2011 June 11 UT using the two-color Differential Speckle Survey Instrument at the Wisconsin Indiana Yale NOAO (WIYN) $3.5 \mathrm{~m}$ telescope, located at the Kitt Peak Observatory (Horch et al. 2009). The speckle camera obtained $700040 \mathrm{~ms}$ images in $I$ band (8880/ $400 \AA$ ). We reduced and processed these observations to produce a final reconstructed speckle image for the star. Details of the speckle camera observations for the Kepler follow-up observing program, including the reduction methods, are presented in Howell et al. (2011).

The speckle observations allow detection of a companion star within the approximately $2.76 \times 2.76$ arcsec box centered on the target. We can detect, or rule out, companions between 0.05 arcsec and 1.8 arcsec from Kepler-61 and, in this case, we did not detect a companion star. We report the limiting
Table 3

Magnitude Limits on Companions to Kepler-61 from Speckle Imaging

\begin{tabular}{lc}
\hline \hline $\begin{array}{l}\text { Radius of Annulus around Kepler-61 } \\
\text { [arcsec] }\end{array}$ & $\begin{array}{r}\text { Limiting Delta Magnitude } \\
3 \sigma \text { Confidence }\end{array}$ \\
\hline $0.05-0.30$ & 2.69 \\
$0.30-0.50$ & 3.05 \\
$0.50-0.70$ & 3.09 \\
$0.70-0.90$ & 3.16 \\
$0.90-1.10$ & 3.15 \\
$1.10-1.30$ & 3.11 \\
$1.30-1.50$ & 3.18 \\
$1.50-1.70$ & 3.24 \\
$1.70-1.90$ & 3.20 \\
\hline
\end{tabular}

difference in magnitude for an additional star that would have been detectable with $3 \sigma$ confidence in Table 3 . The companion detected with AO imaging, which we describe in the previous section, lies just beyond detectability with speckle imaging in the $I$ band, at a distance of $0^{\prime \prime} 94$ and $\Delta m$ in $K$ band $=2.9$ ( 0.25 mag from what would have been detected in the speckle image with $3 \sigma$ confidence at that distance from the star).

\subsection{Photocenter Tests}

We use two methods to search for false positives due to background eclipsing binaries, based on examination of the pixels in the aperture of Kepler-61: direct measurement of the source location via difference images, and inference of the source location from photocenter motion associated with the transits. We employ two methods because of their different vulnerabilities to systematic bias; when the methods agree, we have increased confidence in their result.

Difference image analysis (Torres et al. 2013) takes the difference between average in-transit pixel images and average out-of-transit images. A fit of the Kepler pixel response function (Bryson et al. 2010) to both the difference and out-of-transit images directly provides the location of the transit signal relative to the host star. We measure difference images separately in each quarter, and estimate the transit source location as the robust uncertainty-weighted average of the quarterly results.

We measure photocenter motion by computing the fluxweighted centroid of the pixels in the optimal aperture, plus 
a one-pixel halo in every cadence, generating a centroid time series for row and column. We fit the modeled transit to the whitened centroid time series transformed into sky coordinates. We perform a single fit for all quarters, and then infer the source location by scaling the difference of these two centroids by the inverse of the flux as described in Jenkins et al. (2010a).

The source as determined by the difference image method is offset from the nominal location of Kepler-61, as given in the $\mathrm{KIC}$, by $0.09 \pm 0.29 \operatorname{arcsec}=0.68 \sigma$. The source as determined by the flux-weighted centroid method is offset from Kepler- 61 by $0.32 \pm 0.37$ arcsec $=0.86 \sigma$. Both methods show that the observed centroid location is consistent with the transit occurring at the location of Kepler-61, and rule out the companion in the AO imaging as the source of the transit, which is $3 \sigma$ removed from position at which the transit occurs.

\subsection{Spitzer Observations}

Warm Spitzer observations in the near-infrared can also prove useful toward validating Kepler candidates, as shown for Kepler-10c (Fressin et al. 2011), Kepler-14b (Buchhave et al. 2011), Kepler-18c and d (Cochran et al. 2011), Kepler-19b (Ballard et al. 2011), Kepler-22b (Borucki et al. 2011), Kepler-25b and c (Steffen et al. 2012), and Kepler-20c, d, e, and f (Gautier et al. 2012; Fressin et al. 2012). Unless a putative blend scenario is comprised of stars of nearly identical color, the transit depth in a blend scenario will depend upon the wavelength at which it is observed. Conversely, an authentic transiting planet will produce an near-achromatic transit depth.

We gathered observations using the Infrared Array Camera (IRAC; Fazio et al. 2004) on Warm Spitzer at $4.5 \mu \mathrm{m}$ of the UT 2011 September 17 transit of Kepler-61b. The observations span $10 \mathrm{hr}$, centered on the $4.75 \mathrm{hr}$ long transit. We gathered the observations using the full-array mode of IRAC, with an integration time of $12 \mathrm{~s}$ /image. We employed the techniques described in Agol et al. (2010) for the treatment of the images before photometry. We first converted the Basic Calibrated Data products from the Spitzer IRAC pipeline (which applies corrections for dark current, flat field variations, and detector non-linearity) from mega-Janskys per steradian to data number per second, using $0.1469 \mathrm{MJy} \mathrm{sr}^{-1}$ per $\mathrm{DN} \mathrm{s} \mathrm{s}^{-1}$, and then to electrons per second, using the gain of $3.71 e \mathrm{DN}^{-1}$. We identified cosmic rays by performing a pixel-by-pixel median filter, using a window of 10 frames. We replace pixels that are $>4 \sigma$ outliers within this window with the running median value. We also corrected for a striping artifact in the Warm Spitzer images, which occurred in the same set of columns, by taking the median of the pixel values in the affected columns (using only rows without an overlying star) and normalizing this value to the median value of neighboring columns.

We discuss several means of performing the Warm Spitzer IRAC photometric reduction for similar observations in Ballard et al. (2011), and make use of the conclusions from that work. First, we estimate the position of the star on the array with a flux-weighted sum of the position within a circular aperture of 3 pixels. We then performed aperture photometry on the images using the centroid positions and variable aperture sizes between 2.1 and 4.0 pixels, in increments of 0.1 pixels up to 2.7 pixels, and then at 3.0 and 4.0 pixels. We decided to use the position estimates using a flux-weighted sum at an aperture of 2.6 pixels, which minimized the out-of-transit RMS.

We remove the effect of the IRAC intrapixel sensitivity variations, or the "pixel-phase" effect (see, e.g., Charbonneau et al. 2005; Knutson et al. 2008) using a polynomial functional form for the intrapixel sensitivity (which depends upon the $x$ and $y$ position of the star on the array). We denote the transit light curve $f$ (which depends upon time), and we hold all parameters constant except for the transit depth. We use the light curve software of Mandel \& Agol (2002) to generate the transit models. The model for the measured brightness $f^{\prime}(x, y)$ is given by

$$
\begin{aligned}
f^{\prime}= & f\left(t, R_{p} / R_{\star}\right) \cdot\left[b_{1}+b_{2}(x-\bar{x})\right. \\
& \left.+b_{3}(x-\bar{x})^{2}+b_{4}(y-\bar{y})+b_{5}(y-\bar{y})^{2}\right],
\end{aligned}
$$

where we include all of the observations (both in- and out-oftransit) to fit the polynomial coefficients and the transit depth simultaneously.

We fit for the polynomial coefficients $b_{1}$ through $b_{5}$ using a Levenberg-Marquardt $\chi^{2}$ minimization. However, the Spitzer light curve contains significant correlated noise even after the best intrapixel sensitivity model is removed. We incorporate the effect of remaining correlated noise with a residual permutation analysis of the errors as described by Winn et al. (2008), wherein we find the best-fit model $f^{\prime}$ to the light curve as given by Equation (6), subtract this model from the light curve, shift the residuals by one data point in time, add the same model back to the residuals, and refit the depth and pixel sensitivity coefficients. We wrap residuals from the end of the light curve to the beginning, and in this way we cycle through every permutation of the data. We determine the best value from the median of this distribution, and estimate the error from the closest $68 \%$ of values to the median. Using the residual permutation method on the light curve treated with a polynomial, we find $R_{p} / R_{\star}=0.0315 \pm 0.0069$, in excellent agreement with the Kepler measurement of $R_{p} / R_{\text {star }}=0.03476 \pm 0.00094$.

We note that the use of the weighted sensitivity function proposed in Ballard et al. (2010) made a negligible difference to the photometric residuals in this case, so for reasons of computational time, we deferred to the polynomial reduction technique. In Figure 10, we show the combined and binned Spitzer light curve, with the best-fit transit model derived from the Spitzer observations and the best-fit Kepler transit model (with the quadratic LDCs for the Spitzer $4.5 \mu \mathrm{m}$ filter, drawn from Claret \& Bloemen 2011 as similarly described in Section 1) overplotted.

\subsection{BLENDER Validation}

Traditional confirmation of the planetary nature of a transit signal relied upon a dynamical mass measurement from radial velocity observations. In cases where the reflex motion induced on the host star by the planet is too small to be detected, dynamical confirmation may yet be possible via transit timing variations. However, in cases where a dynamical mass measurement via either technique is not viable, it is still possible to "validate" the planetary nature of the transit signal, via a statistical argument about the relative likelihood of an authentic planet producing the transit signal, as compared to a false positive scenario. BLENDER is such a machinery, which combines evidence from the Kepler photometry (as compared to model light curves for planetary and false positive scenarios), spectroscopy, Spitzer photometry (where available), the stellar colors, and AO to deduce a false positive probability for a planetary candidate. BLENDER has already been applied to validate planets in a number of Kepler exoplanetary systems, including CoRoT-7 (Fressin et al. 2012), Kepler-10 Fressin et al. (2011), Kepler-18 (Cochran et al. 2011), Kepler-19 (Ballard et al. 2011), 

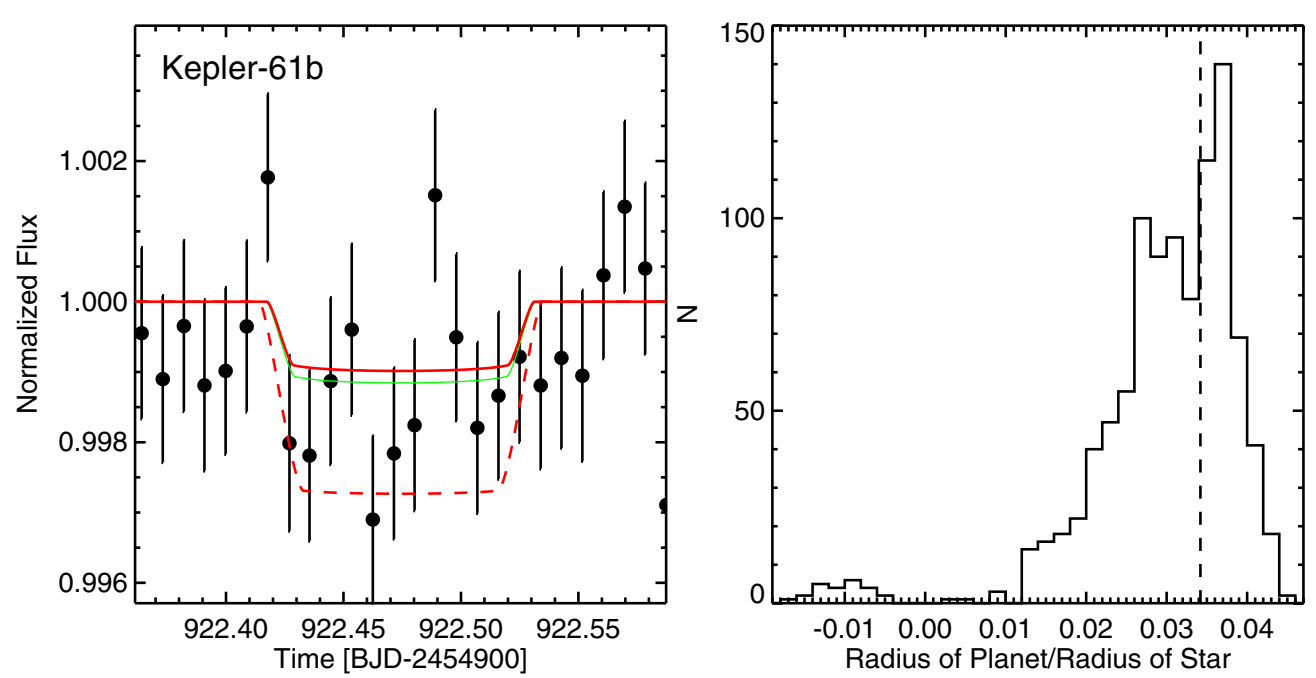

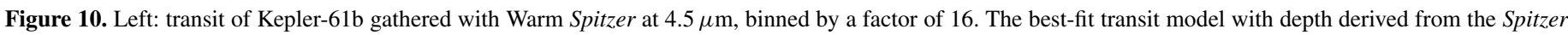

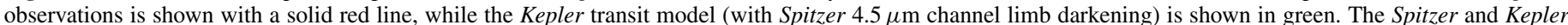

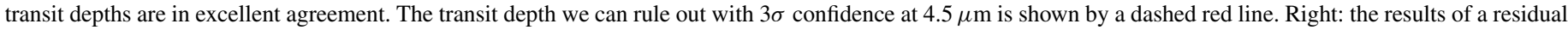

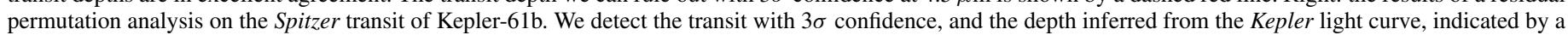
dotted line, lies within one standard deviation of the depth inferred from Spitzer.

(A color version of this figure is available in the online journal.)

Kepler-20 (Gautier et al. 2012), Kepler-21 (Howell et al. 2012), and Kepler-22 (Borucki et al. 2012), and its details are described therein as well as in Torres et al. (2004), Fressin et al. (2011), and Fressin et al. (2012). We summarize the BLENDER procedure below, and present the statistical likelihood that the transit signal presented by Kepler-61b is attributable to a $2.5 R_{\oplus}$ planet orbiting a $0.65 R_{\odot}$ star.

The exquisite precision of the Kepler photometry is already sufficient to rule out some false-positive scenarios, which would produce a significantly different transit shape from the one observed by Kepler. Such a false positive could mimic the observed transit depth if an additional star fell within the same aperture of the Kepler target star. The light contributed by this undetected companion (which may be gravitationally bound to the target star or lie in the foreground or background), would reduce the transit depth produced by an eclipsing binary system or a planetary system comprising a star and a larger planet, conspiring to produce a planetary transit depth. BLENDER manufactures synthetic light curves corresponding to these false positive scenarios: for those with a physically bound companion, BLENDER assumes a common age for the putative companion star and the Kepler target star, while an unassociated background or foreground star is assigned an age of 3 Gyr. The mass of this secondary star and the tertiary body (either star or planet) is allowed to vary. These model blend light curves are compared to the Kepler photometry in a $\chi^{2}$ sense. Blend scenarios that furnish a good fit to the Kepler light curve (within $3 \sigma$ of the best authentic planet model) are then tested for consistency against other constraints. These include (1) the color of the star as reported in the KIC (Brown et al. 2011), which allows us to rule out any simulated blends resulting in a combined color that is significantly redder or bluer than the target; (2) limits from the centroid motion analysis on the angular separation of companions that could produce the signal (Section 4.3); (3) brightness and angular separation limits from high-resolution AO (Section 4.1); and (4) constraints from the measured transit depth derived from our Spitzer observations, which place an upper limit on the mass (spectral type) of stars producing the blend. For the hierarchical triple scenario (in which the secondary star is physically bound to the Kepler target star), we considered dynamical stability constraints (Holman $\&$ Wiegert 1999). Surviving blend scenarios that satisfy all of the above criteria are folded into the "blend frequency": which is the probability that such a finely tuned blend lies near enough to the target star to be undetectable by AO imaging (using the sensitivity limits we find in Section 4.1). We compared this frequency with the expected frequency of true planets (the planet "prior") to derive the "odds ratio." To estimate the planet prior, we employ the list of candidate planets (KOIs) from Batalha et al. (2013), restricted to main-sequence host stars. We assume that this list is complete (i.e., that all signals have been detected) and that the rate of false positives is negligible (which assumption is justified by the findings of Morton \& Johnson 2011, who found a false-positive rate of $<5 \%$ for most KOIs).

We find that background eclipsing binaries comprising two stars furnish only poor fits to the Kepler photometry, and so are excluded from Kepler data alone. A portion of the hierarchical triple parameter space (with a star and larger planet gravitationally bound to the Kepler target star) provides good fits to the Kepler photometry, but these scenarios are then excluded by the combination of observational constraints described above. Background stars transited by larger planets, on the other hand, can mimic the Kepler photometry and remain consistent with the Kepler centroid motion, follow-up AO imaging, spectroscopy, and Spitzer constraint. We find that the frequency of background/foreground blends that satisfy these criteria is $4.19 \times 10^{-8}$. The planet prior is estimated by counting the number of known KOIs that are in the same $(3 \sigma)$ radius range as the putative planet (105 in this case), and dividing by the total number of main-sequence Kepler targets observed during Q1-Q6 $(138,253)$. We obtained a planet prior of $105 / 138,253=7.60 \times 10^{-4}$. We conclude that a true transiting planet is $7.60 \times 10^{-4} / 4.19 \times 10^{-8}=18,000$ times more likely than a blend, which allows us to validate Kepler-61 with a high degree of confidence. 
The simple procedure described above for estimating the planet prior does not take into account the period of the signal, which may be an important factor for small and long-period candidates (such as Kepler-61b) because such signals are rare (see below). Furthermore, the completeness and purity of the KOI list of Batalha et al. (2013), on which our planet prior calculation relies, may decline with period and planetary radius, whereas we have assumed these concerns are negligible. These factors may in principle influence both the planet prior and the blend frequencies we have just described (since we employ the KOI list not only to estimate the occurrence of authentic small planets, but also to estimate the rate of occurrence of larger planets in false-positive blend scenarios). Therefore, instead of allowing eclipsing binaries and transiting planets with any orbital period to factor into the blend frequency calculation, we elected to redo the BLENDER analysis with a more realistic approach to allowed blends. First, we accept only blends with periods near the measured periods of Kepler-61 (within a factor of two) for both the blend and planet prior calculation. To address the concerns about completeness and purity of the KOI list, we performed separate Monte Carlo simulations to establish incompleteness corrections for the KOI list and also to estimate the false positive rates for planets in the size ranges relevant to this calculation. A description of this work is forthcoming (Fressin et al. 2013). We obtained a revised frequency of background/foreground blends of $7.27 \times 10^{-9}$. Examining the candidate list of Batalha et al. (2013), we found 22 KOIs in the relevant radius range with periods within a factor of two of the period of Kepler-61. Our simulations suggest that about 2.09 of these may be false positives, but also that the KOI list for signals of this size and period is in fact incomplete, requiring a correction factor of approximately 1.59 (i.e., a signal such as that of Kepler-61 could only have been detected around 63\% of main-sequence Kepler targets). The corrected planet count is then $(22-2.09) \times 1.59=31.66$. With this, the planet prior becomes $31.66 / 138,253=2.29 \times 10^{-4}$. The final odds ratio for Kepler-61b is then $2.29 \times 10^{-4} / 7.27 \times 10^{-9}=31,500$, which 1.75 times more significant as we found with a more simplified approach.

Blends that include a companion star $<0.45 M_{\odot}$ would produce transit depths inconsistent with our Spitzer observations, since they would produce transits depths more than $3 \sigma$ deeper than we measure at $4.5 \mu \mathrm{m}$. These blends are thus excluded. For Kepler-61, the Spitzer results exclude all remaining possible physically bound configurations, which would have been the major cause of false positives otherwise. We depict this constraint in the bottom panel of Figure 11.

We note that we have assumed that any signal with a signalto-noise ratio larger than 7.1 would have been recovered by the Kepler pipeline as a KOI, to compute the incompleteness correction factor. This optimistic hypothesis is a conservative one in our case, since a more realistic detection model would further increase the incompleteness correction to our planet prior. We conclude that Kepler-61 is an authentic $2.15 R_{\oplus}$ planet with a high degree of confidence. We depict an illustration of the BLENDER constraints on false positives for Kepler-61 in Figure 11.

\section{DISCUSSION AND CONCLUSIONS}

\subsection{Transit Times}

We depict the transit times of Kepler-61b in Figure 12. We report no significant deviation from a linear ephemeris.
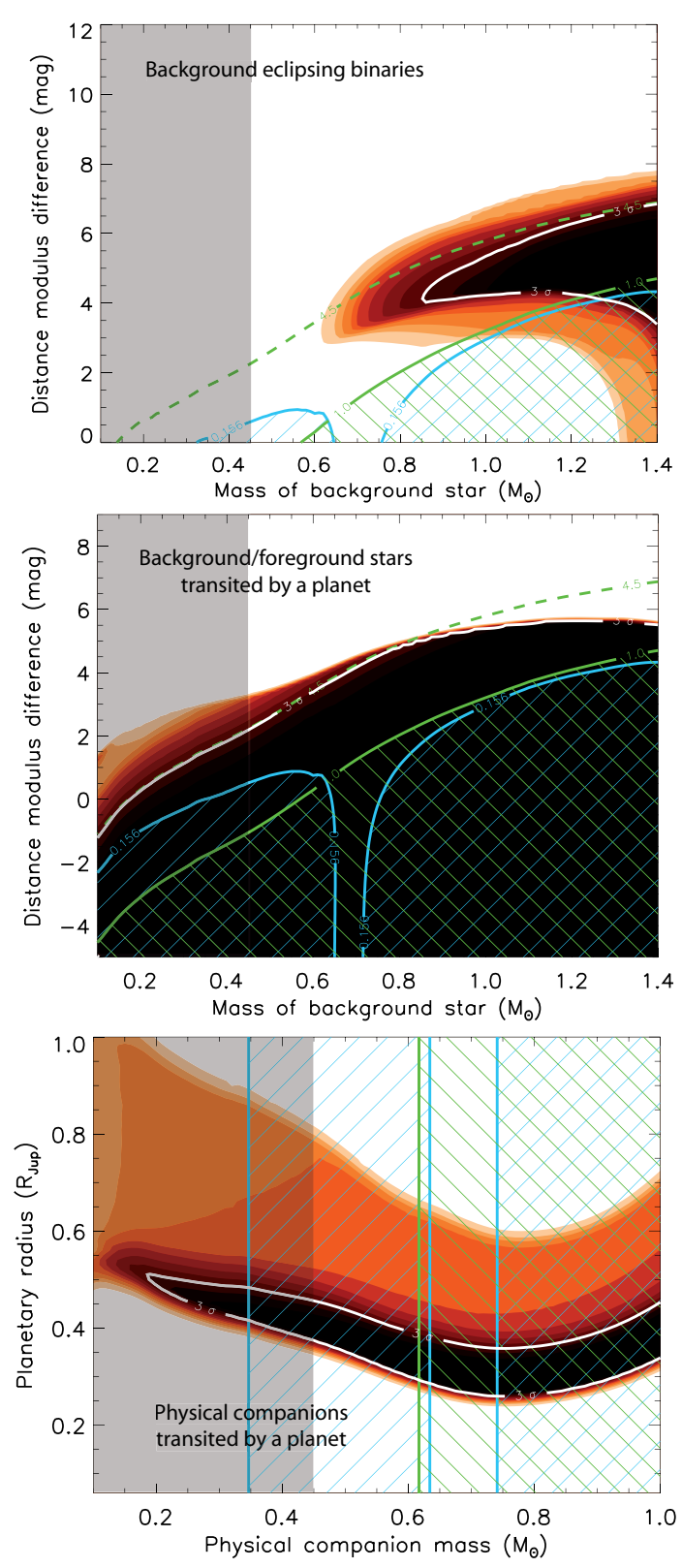

Figure 11. BLENDER goodness-of-fit contours for Kepler-61b corresponding to the three different scenarios that contribute to the overall blend frequency: background eclipsing binaries (top), background or foreground stars transited by a planet (middle), and physical companions transited by a planet (bottom). Solid colored contours depict the difference in $\chi^{2}$ between an authentic transit model and a blend fit with those parameters. Only blends within the solid white contour acceptably match the Kepler light curve ( $3 \sigma$ difference in $\chi^{2}$ between the blend and transit model fit (see Fressin et al. 2011), while red, orange, and yellow contours correspond to blend models disfavored by the Kepler photometry by $4 \sigma, 5 \sigma$, and $6 \sigma$, respectively. The axes in each panel represent two of the dimensions of parameter space for blends. For the top two diagrams the vertical axis depicts the distance modulus difference between the two stars, while the horizontal axis corresponds to the mass (spectral type) of the putative secondary star. In the bottom panel (hierarchical triple scenario), the vertical axis corresponds the size of the planet transiting the companion star. The cyan cross-hatched areas indicate regions of parameter space ruled out because the resulting Sloan $r^{\prime}$-2MASS $K$ color of the blend is either too red (left) or too blue (right) compared to the measured color, by more than $3 \sigma(0.15 \mathrm{mag})$. The green hatched regions indicate blends that are ruled out because the additional star is less than 1 mag fainter than the target and would have been detected spectroscopically. Finally, the gray areas on the left represents the constraint from our Spitzer observations. The diagonal dashed green lines in the top two panels indicate the faintest blends that can mimic the transit: approximately $\Delta \mathrm{Kp}=4.5 \mathrm{mag}$ both for background eclipsing binaries and for background/ foreground stars transited by a planet.

(A color version of this figure is available in the online journal.) 


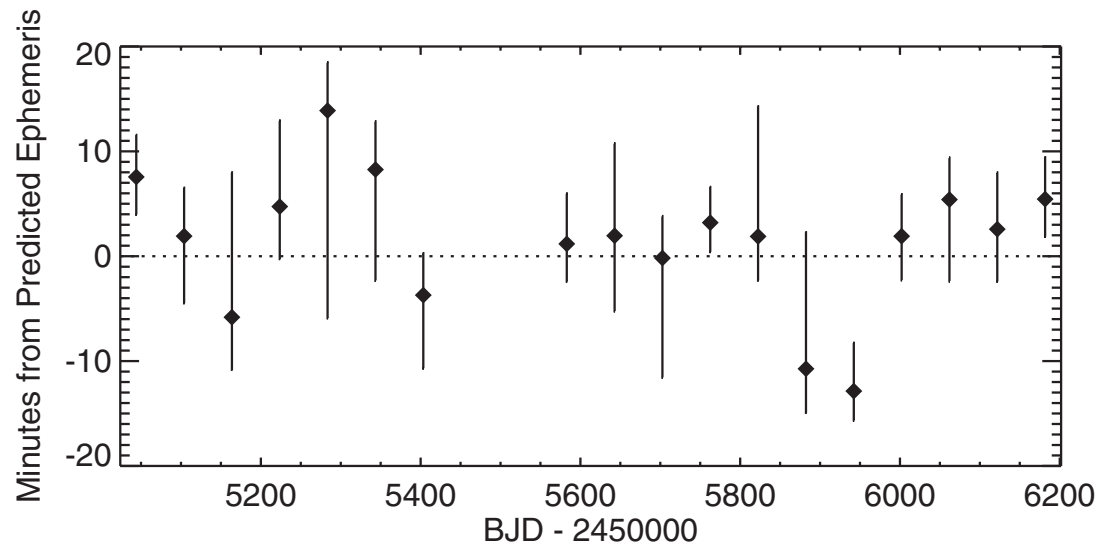

Figure 12. Kepler transit times for Kepler-61 from Quarters 1-14, as compared to the best linear ephemeris model.

Table 4

Properties of Transiting Planets from 1.4-3.0 $R_{\oplus}$ with Dynamically Measured Masses

\begin{tabular}{lcccc}
\hline \hline Name & $\begin{array}{c}\text { Radius } \\
\left(R_{\oplus}\right)\end{array}$ & $\begin{array}{c}\text { Mass } \\
\left(M_{\oplus}\right)\end{array}$ & $\begin{array}{c}\text { Mean Density } \\
\left(\mathrm{g} \mathrm{cm}^{-3}\right)\end{array}$ & Reference \\
\hline Kepler-68c & $0.953_{-0.042}^{+0.037}$ & $4.8_{-3.6}^{+2.5}$ & $28_{-23}^{+13}$ & Gilliland et al. (2013) \\
Kepler-10b & $1.416_{-0.036}^{+0.033}$ & $4.56_{-1.29}^{+1.17}$ & $8.8_{-2.9}^{+2.1}$ & Batalha et al. (2011) \\
Kepler-36b & $1.486 \pm 0.035$ & $4.45_{-0.27}^{+0.33}$ & $7.46_{-0.59}^{+0.74}$ & Carter et al. (2012) \\
CoRoT-7b & $1.58 \pm 0.10$ & $7.42 \pm 1.21$ & $10.4 \pm 1.8^{\mathrm{a}}$ & Bruntt et al. (2010), \\
& & & & Hatzes et al. (2011) \\
Kepler-20b & $1.91_{-0.21}^{+0.12}$ & $8.7 \pm 2.2$ & $6.9_{-2.6}^{+5.3 \mathrm{~b}}$ & Gautier et al. (2012) \\
Kepler-11b & $1.97 \pm 0.19$ & $4.3_{-2.0}^{+2.2}$ & $3.1_{-1.5}^{+2.1}$ & Lissauer et al. (2011) \\
Kepler-18b & $2.00 \pm 0.10$ & $6.9 \pm 3.4$ & $4.9 \pm 2.4$ & Cochran et al. (2011) \\
55 Cnc e & $2.00 \pm 0.14$ & $8.63 \pm 0.35$ & $5.9_{-1.1}^{+1.5}$ & Winn et al. (2011) \\
Kepler-68b & $2.31 \pm 0.07$ & $8.3 \pm 2.3$ & $3.32 \pm 0.92$ & Gilliland et al. (2013) \\
Kepler-11f & $2.61 \pm 0.25$ & $2.3_{-1.2}^{+2.2}$ & $0.7_{-0.4}^{+0.7}$ & Lissauer et al. (2011) \\
GJ 1214b & $2.678 \pm 0.13$ & $6.55 \pm 0.98$ & $1.87 \pm 0.4$ & Charbonneau et al. (2009)
\end{tabular}

Notes.

a Differing mass measurements of CoRoT-7b (Queloz et al. 2009; Pont et al. 2011) furnish different mean densities; we have stated the most recently published values.

${ }^{\mathrm{b}}$ Mean density calculated from stated $1 \sigma$ limits in radius and mass.

\subsection{Theoretical Composition of Kepler-61b}

\subsubsection{Bulk Composition and Atmosphere}

While we cannot estimate the mean density of Kepler-61b without a measurement of its mass, we can still discuss plausible compositions, given its equilibrium temperature and radius. There now exist a sizable set of exoplanets with radii in the 1.0-3.0 $R_{\oplus}$ range with dynamically measured masses, though these span a large range of bulk densities from $0.7 \mathrm{~g} \mathrm{~cm}^{-3}$ in the case of Kepler-11f to $10.4 \mathrm{~g} \mathrm{~cm}^{-3}$ in the case of CoRoT-7b (Kepler-68c may comprise a very dense exception, with $\rho=28_{-23}^{+13}$, but the density range is large and relatively unconstraining). We list published masses, radii, and mean densities from the literature in Table 4. The two planets nearest to Kepler-61 in radius are 55 Cancri e (Winn et al. 2011) and Kepler-68b (Gilliland et al. 2013), the radii of which lie within $0.15 R_{\oplus}$ of the radius of Kepler-61. Carter et al. (2012) found that, even within the small known sample of super-Earths with measured radii, a trend is apparent for planets with equilibrium temperatures $<1200 \mathrm{~K}$ : these tend to have "mini-Neptune" compositions, with mean density $<3.5 \mathrm{~g} \mathrm{~cm}^{-3}$. However, these planets (Kepler-11b, d, e, f, and g, described by Lissauer et al. 2011, and GJ 1214b, described by Charbonneau et al. 2009) are also near or larger than $2 R_{\oplus}$, whereas all planets with measured densities higher than approximately $7 \mathrm{~g} \mathrm{~cm}^{-3}$ have radii smaller than $2 R_{\oplus}$. It is therefore unclear whether the low density of this set of cooler planets is attributable to their planetary radius or their insolation, or both; this question is explored in greater detail in Weiss et al. (2013), who incorporated both quantities in relation to planetary mass in their exoplanetary mass-radius relation.

We also consider the theoretical atmospheric content of a $2.15 R_{\oplus}$ planet. Rogers et al. (2011) consider two scenarios (core accretion and outgassing) by which planets in the $2-4 R_{\oplus}$ regime might retain a substantial hydrogen and helium envelope. Though that work focuses on temperatures $>500 \mathrm{~K}$, a cooler temperature would extend still longer the lifetime of a putative hydrogen/helium envelope. For example, if Kepler-61 formed by core-nucleated accretion beyond the snow line, at $500 \mathrm{~K}$ (substantially warmer), a hydrogen helium envelope fraction of $0.1 \%$ by mass is plausible for timescales $<1$ Gyr. Alternatively, it the hydrogen content of the atmosphere is outgassed from the planet (assumed to be formed from iron enstatite), a mass fraction of $1 \%$ by mass is plausible for timescales as long as $100 \mathrm{Gyr}$. These timescales (and their corresponding atmospheric mass fractions) should be considered lower bounds, given these 
formation scenarios, since a cooler planet like Kepler-61 will retain an atmosphere for a longer duration, all else being equal. The synthetic planetary radius distribution generated by the formation models of Mordasini et al. (2012), which assume a primordial hydrogen/helium envelope, furnishes a good match to the Kepler candidates for planets larger than $2 R_{\oplus}$, but diverges from the Kepler results for smaller radii. This result may be attributable to the more terrestrial composition of planets smaller than $2 R_{\oplus}$, for which the assumption of a hydrogen/ helium envelope is no longer valid (Mordasini et al. 2012).

We conclude that a density larger than $7 \mathrm{~g} \mathrm{~cm}^{-3}$, which has only be observed for planets $<2 R_{\oplus}$, is unlikely for Kepler61 . Given its radius and comparatively low stellar insolation, its mass may be closer to the $3-6 \mathrm{~g} \mathrm{~cm}^{-3}$ density range bracketed by $55 \mathrm{Cnc}$ e or Kepler-68b with similar radii. If we apply the relation derived by Weiss et al. (2013) from the sample of exoplanets with radius measurements and masses $<150 M_{\oplus}$ (another power law applies for more massive planets), we find a predicted mass and density for Kepler- $61 \mathrm{~b}$ of $3.2 M_{\oplus}$ and $2.4 \mathrm{~cm}^{-3}$, respectively, near that of Kepler-11b (Lissauer et al. 2011).

There also exist theoretical constraints on the sustainability of super-Earth atmospheres for higher mean molecular weights. In particular, Heng \& Kopparla (2012) consider the stability of high mean molecular weight atmospheres belonging to super Earths orbiting low-mass stars in particular. The proximity of the habitable zone to the star means that many super Earths will be spin-synchronized, with a permanent day and night side. In particular, the timescale for spin synchronization is given by Bodenheimer et al. (2001) and stated in terms of orbital frequency $\Omega$ by Heng \& Kopparla (2012):

$$
t_{\mathrm{syn}}=\frac{8 Q}{45 \Omega}\left(\frac{\omega}{\Omega}\right)\left(\frac{M_{p}}{M_{\star}}\right)\left(\frac{a}{R_{p}}\right)^{3},
$$

where the planet's initial rotational frequency is given by $w$, $Q$ is the tidal quality factor (and is believed to lie within the range of 10-100 for rocky exoplanets, and in the $10^{5}-10^{6}$ range for gas giants, as stated in Goldreich \& Soter 1966). Even with extremely rapid initial rotational periods of the planet (e.g., 0.1 day) and values for $Q$ which approach that of gas giants, Kepler-61 is close enough to its host star where the spin synchronization timescale is less than $1 \mathrm{Myr}$.

This poses a problem for atmospheric stability unless the zonal winds' ability to redistribute heat in the atmosphere outstrips the radiative timescale of the atmosphere. If this condition (namely, that the advective timescale is shorter than the radiative timescale) does not hold, then the low temperature of "night side" of the planet can allow heavier elements to condense out, leaving the atmosphere unstable. An atmosphere comprising heavier elements has a longer advective timescale, since the wind speed is slowed as mean molecular weight increases (similarly to the sound speed). For this reason, Earthlike atmospheres (with mean molecular weights of 30) are particularly susceptible to instability. The fact that Kepler-61 orbits a late $\mathrm{K}$ dwarf translates to a radiative longer than the advective timescale (Heng \& Kopparla 2012), so an Earth-like atmosphere would remain stable. For later M stars (for example, an M 3.5V star, as adopted as a trial case by Heng \& Kopparla 2012), the radiative timescale at $0.25 \mathrm{AU}$ is shorter, so a $2.5 R_{\oplus}$ planet possessing an atmosphere with mean molecular weight of 30 would be potentially unstable.

\subsection{Future Prospects}

We comment briefly on the feasibility of atmospheric characterization of Kepler-61b. It orbits a small star and may possess a hydrogen and helium atmosphere, both of which are favorable circumstances for transmission spectroscopy. To perform a basic estimation of the expected change in transit depth at an optically thick wavelength, we consider the atmosphere to be a ring with scale height $H$, where $H=k T_{p} / \mu_{m} g$ (and $k$ is Boltzmann's constant, $T_{p}$ is the temperature of the planet, $\mu_{m}$ is the mean molecular weight of the atmosphere, and $g$ is the surface gravity of the planet). If we use a mass estimate of $8 M_{\oplus}$ for Kepler- $61 \mathrm{~b}$ (near that measured by Gilliland et al. 2013 for Kepler-68b, with a similar radius), then we expect a surface gravity of $17 \mathrm{~m} \mathrm{~s}^{-2}$. If we assume the most optimistic case from a detectability standpoint, we also use molecular weight of 2 (corresponding to the hydrogen-rich scenario). Employing the same equation to estimate the change in transit depth attributable to the atmosphere as Miller-Ricci et al. (2009), we expect a change in transit depth given by

$$
\Delta D \approx \frac{2 \pi R_{p} H}{\pi R_{\star}^{2}}=\frac{2 R_{p} H}{R_{\star}^{2}},
$$

which equates to $\Delta D=10 \mathrm{ppm}$, if we employ the values for $R_{p}$ and $R_{\star}$ given in Table 2. If we instead assume a mass of $2.3 M_{\oplus}$, like that of the $2.6 R_{\oplus}$ planet Kepler-11f (Lissauer et al. 2011), then $\Delta D$ is correspondingly three times larger, at $30 \mathrm{ppm}$. This signal is approximately one-tenth the size of the $0.5 \mathrm{mmag}$ values which might have been detectable in the atmosphere of GJ 1214b by Berta et al. (2012) using the Wide Field Camera 3 on board the Hubble Space Telescope. However, Kepler-61 is also 25 times dimmer in $K$ band than GJ 1214, rendering the detection of an atmosphere around Kepler-61 out of the reach of current instruments.

Similarly, the radial velocity amplitude of Kepler-61b is increased by the small mass of the host star. In this case, assuming again a mass of $8 M_{\oplus}$ for the planet and a mass of $0.64 M_{\odot}$ for the star, the planet induces a $1.8 \mathrm{~m} \mathrm{~s}^{-1}$ motion of its star. However, though measuring a radial velocity signature of several meters-per-second has been achieved for dozens of exoplanets, these are all around very nearby stars. Kepler-61b, with its Kepler magnitude $\mathrm{Kp}$ of 15.0 , is probably too dim for such study with current instruments. However, gathering additional Kepler observations of Kepler-61b will be helpful, particularly given the fact that it will be observed in short cadence mode for Quarter 12 onward.

\subsection{Conclusions}

We present the validation and characterization of Kepler-61b, a $2.15 \pm 0.13 R_{\oplus}$ exoplanet with an equilibrium temperature of $273 \pm 13 \mathrm{~K}$, orbiting a late $\mathrm{K}$ dwarf. We determine that the planetary hypothesis for the transit signature of Kepler-61b is 30,000 times more likely than the false positive hypothesis, folding together evidence from high-resolution imagery, the stellar colors, the centroid position of the star from the Kepler images, the depth of the transit in the $4.5 \mu \mathrm{m}$ bandpass from Spitzer, and from the detailed comparison of the Kepler photometry to theoretical light curves of both planetary transits and stellar blends. Our measurement of the radius and temperature of the star Kepler-61 is based upon a weighted mean of the directly measured radii and temperatures of a subset of nearby stars with the same spectral type, which quantities we apply as priors in our characterization of the planet. We present $K$-band spectra and newly derived metallicities for this set of four similar 
stars, as well as for Kepler-61. The application of this empirical method, as compared to characterization from $K$-band spectra and stellar evolutionary models, ultimately increased the size and temperature of the planet by $10 \%$. We consider plausible compositions for Kepler- $61 \mathrm{~b}$ from the set of planets with similar radii and dynamically measured masses, as well as from massradius relationships for exoplanets. We conclude that the planet is likely slightly too large to be terrestrial in composition, and likely possesses a significant atmosphere.

We thank Perry Berlind and Mike Calkins at the Fred Lawrence Whipple Observatory for gathering the FAST spectra of Kepler-61 and GJ 380. We thank Courtney Dressing for applying the methodology of Dressing \& Charbonneau (2013) to deduce the physical properties of Kepler-61 and sharing these values with us. We thank Philip Muirhead, Katherine Hamren, Everett Schlawin, Bárbara Rojas-Ayala, Kevin Covey, and James Lloyd for gathering, reducing, and sharing the TripleSpec $K$-band spectrum of Kepler-61. We thank the Spitzer team at the Infrared Processing and Analysis Center in Pasadena, California, and in particular Nancy Silbermann for scheduling the Spitzer observations of this program. This work was performed in part under contract with the California Institute of Technology (Caltech) funded by NASA through the Sagan Fellowship Program. It was conducted with observations made with the Spitzer Space Telescope, which is operated by the Jet Propulsion Laboratory, California Institute of Technology under a contract with NASA. Support for this work was provided by NASA through an award issued by JPL/Caltech. This work is also based on observations made with Kepler, which was competitively selected as the tenth Discovery mission. Funding for this mission is provided by NASA's Science Mission Directorate. The authors would like to thank the many people who generously gave so much their time to make this Mission a success. This research has made use of the NASA Exoplanet Archive, which is operated by the California Institute of Technology, under contract with the National Aeronautics and Space Administration under the Exoplanet Exploration Program.

\section{REFERENCES}

Agol, E., Cowan, N. B., Knutson, H. A., et al. 2010, ApJ, 721, 1861 Agüeros, M. A., Covey, K. R., Lemonias, J. J., et al. 2011, ApJ, 740, 110 Allard, F., Hauschildt, P. H., \& Schwenke, D. 2000, ApJ, 540, 1005 Allard, F., Homeier, D., \& Freytag, B. 2012, RSPTA, 370, 2765 Argabright, V. S., van Cleve, J. E., Bachtell, E. E., et al. 2008, Proc. SPIE, 7010 70102L

Baliunas, S., Sokoloff, D., \& Soon, W. 1996, ApJL, 457, L99

Ballard, S., Charbonneau, D., Deming, D., et al. 2010, PASP, 122, 1341

Ballard, S., Fabrycky, D., Fressin, F., et al. 2011, ApJ, 743, 200

Banfield, D., \& Murray, N. 1992, Icar, 99, 390

Barnes, S. A. 2003, ApJ, 586, 464

Barnes, S. A. 2007, ApJ, 669, 1167

Barnes, S. A. 2010, ApJ, 722, 222

Batalha, N. M., Borucki, W. J., Bryson, S. T., et al. 2011, ApJ, 729, 27

Batalha, N. M., Borucki, W. J., Koch, D. G., et al. 2010, ApJL, 713, L109

Batalha, N. M., Rowe, J. F., Bryson, S. T., et al. 2013, ApJS, 204, 24

Berta, Z. K., Charbonneau, D., Désert, J.-M., et al. 2012, ApJ, 747, 35

Bodenheimer, P., Lin, D. N. C., \& Mardling, R. A. 2001, ApJ, 548, 466

Borucki, W. J., Agol, E., Fressin, F., et al. 2013, Sci, 340, 587

Borucki, W. J., Koch, D. G., Basri, G., et al. 2011, ApJ, 736, 19

Borucki, W. J., Koch, D. G., Batalha, N., et al. 2012, ApJ, 745, 120

Boyajian, T. S., von Braun, K., van Belle, G., et al. 2012, ApJ, 757, 112

Brown, T. M., Charbonneau, D., Gilliland, R. L., Noyes, R. W., \& Burrows, A. 2001, ApJ, 552, 699

Brown, T. M., Latham, D. W., Everett, M. E., \& Esquerdo, G. A. 2011, AJ, 142,112

Browning, M. K. 2008, ApJ, 676, 1262
Bruntt, H., Deleuil, M., Fridlund, M., et al. 2010, A\&A, 519, A51 Bryson, S. T., Tenenbaum, P., Jenkins, J. M., et al. 2010, ApJL, 713, L97 Buchhave, L. A., Latham, D. W., Carter, J. A., et al. 2011, ApJS, 197, 3 Caldwell, D. A., van Cleve, J. E., Jenkins, J. M., et al. 2010, Proc. SPIE, 7731, 773117

Carter, J. A., Agol, E., Chaplin, W. J., et al. 2012, Sci, 337, 556

Casagrande, L., Flynn, C., \& Bessell, M. 2008, MNRAS, 389, 585

Charbonneau, D., Allen, L. E., Megeath, S. T., et al. 2005, ApJ, 626, 523

Charbonneau, D., Berta, Z. K., Irwin, J., et al. 2009, Natur, 462, 891

Claret, A., \& Bloemen, S. 2011, A\&A, 529, A75

Cochran, W. D., Fabrycky, D. C., Torres, G., et al. 2011, ApJS, 197, 7

Covey, K. R., Ivezić, Ž., Schlegel, D., et al. 2007, AJ, 134, 2398

Cutri, R. M., Skrutskie, M. F., van Dyk, S., et al. 2003, yCat, 2246, 0

Dotter, A., Chaboyer, B., Jevremović, D., et al. 2008, ApJS, 178, 89

Dressing, C. D., \& Charbonneau, D. 2013, ApJ, 767, $95>$

Edelson, R. A., \& Krolik, J. H. 1988, ApJ, 333, 646

Fabrycky, D. C., Ford, E. B., Steffen, J. H., et al. 2012, ApJ, 750, 114

Fazio, G. G., Hora, J. L., Allen, L. E., et al. 2004, ApJS, 154, 10

Fressin, F., Torres, G., Charbonneau, D., et al. 2013, ApJ, 766, 81

Fressin, F., Torres, G., Désert, J.-M., et al. 2011, ApJS, 197, 5

Fressin, F., Torres, G., Rowe, J. F., et al. 2012, Natur, 482, 195

Gautier, T. N., III, Charbonneau, D., Rowe, J. F., et al. 2012, ApJ, 749, 15

Gilliland, R. L., Marcy, G. W., Rowe, J. F., et al. 2013, ApJ, 766, 40

Gizis, J. E., Reid, I. N., \& Hawley, S. L. 2002, AJ, 123, 3356

Goldreich, P., \& Soter, S. 1966, Icar, 5, 375

Hatzes, A. P., Fridlund, M., Nachmani, G., et al. 2011, ApJ, 743, 75

Heng, K., \& Kopparla, P. 2012, ApJ, 754, 60

Herter, T. L., Henderson, C. P., Wilson, J. C., et al. 2008, Proc. SPIE, 7014, $70140 X$

Holman, M. J., \& Wiegert, P. A. 1999, AJ, 117, 621

Horch, E. P., Veillette, D. R., Baena Gallé, R., et al. 2009, AJ, 137, 5057

Howell, S. B., Everett, M. E., Sherry, W., Horch, E., \& Ciardi, D. R. 2011, AJ, 142,19

Howell, S. B., Rowe, J. F., Bryson, S. T., et al. 2012, ApJ, 746, 123

Irwin, J., Berta, Z. K., Burke, C. J., et al. 2011, ApJ, 727, 56

Jenkins, J. M., Borucki, W. J., Koch, D. G., et al. 2010a, ApJ, 724, 1108

Jenkins, J. M., Caldwell, D. A., Chandrasekaran, H., et al. 2010b, ApJL, 713, L120

Kaltenegger, L., \& Sasselov, D. 2011, ApJL, 736, L25

Kasting, J. 2011, Joint Meeting of the Exoplanet and Cosmic Origins Program Analysis Groups (ExoPAG and COPAG), 2011 April 26, Baltimore, MD (http://exep.jpl.nasa.gov/exopag/exopagCopagJointMeeting/)

Kervella, P., \& Fouqué, P. 2008, A\&A, 491, 855

Kiraga, M., \& Stepien, K. 2007, AcA, 57, 149

Knutson, H. A., Charbonneau, D., Allen, L. E., Burrows, A., \& Megeath, S. T. 2008, ApJ, 673, 526

Kopparapu, R. K., Ramirez, R., Kasting, J. F., et al. 2013, ApJ, 765, 131

Lane, B. F., Boden, A. F., \& Kulkarni, S. R. 2001, ApJL, 551, L81

Lépine, S., Hilton, E. J., Mann, A. W., et al. 2013, AJ, 145, 102

Lissauer, J. J., Fabrycky, D. C., Ford, E. B., et al. 2011, Natur, 470, 53

Mandel, K., \& Agol, E. 2002, ApJL, 580, L171

Mann, A. W., Brewer, J. M., Gaidos, E., Lépine, S., \& Hilton, E. J. 2013, AJ, 145,52

Miller-Ricci, E., Seager, S., \& Sasselov, D. 2009, ApJ, 690, 1056

Mordasini, C., Alibert, Y., Georgy, C., et al. 2012, A\&A, 547, 112

Morton, T. D., \& Johnson, J. A. 2011, ApJ, 738, 170

Muirhead, P. S., Hamren, K., Schlawin, E., et al. 2012a, ApJL, 750, L37

Muirhead, P. S., Johnson, J. A., Apps, K., et al. 2012b, ApJ, 747, 144

Mullan, D. J., \& MacDonald, J. 2001, ApJ, 559, 353

Murray, C. D., \& Correia, A. C. M. 2010, in Keplerian Orbits and Dynamics of Exoplanets, ed. S. Seager (Tucson, AZ: Univ. Arizona Press), 15

Pinsonneault, M. H., An, D., Molenda-Żakowicz, J., et al. 2012, ApJS, 199, 30

Pont, F., Aigrain, S., \& Zucker, S. 2011, MNRAS, 411, 1953

Queloz, D., Bouchy, F., Moutou, C., et al. 2009, A\&A, 506, 303

Rayner, J. T., Cushing, M. C., \& Vacca, W. D. 2009, ApJS, 185, 289

Rayner, J. T., Toomey, D. W., Onaka, P. M., et al. 2003, PASP, 115, 362

Roeser, S., Demleitner, M., \& Schilbach, E. 2010, AJ, 139, 2440

Rogers, L. A., Bodenheimer, P., Lissauer, J. J., \& Seager, S. 2011, ApJ, 738,59

Rojas-Ayala, B., Covey, K. R., Muirhead, P. S., \& Lloyd, J. P. 2012, ApJ, 748,93

Seager, S., \& Mallén-Ornelas, G. 2003, ApJ, 585, 1038

Ségransan, D., Kervella, P., Forveille, T., \& Queloz, D. 2003, A\&A, 397, L5

Smith, J. C., Stumpe, M. C., van Cleve, J. E., et al. 2012, PASP, 124, 1000

Socrates, A., Katz, B., Dong, S., \& Tremaine, S. 2012, ApJ, 750, 106

Sozzetti, A., Torres, G., Charbonneau, D., et al. 2007, ApJ, 664, 1190

Steffen, J. H., Fabrycky, D. C., Ford, E. B., et al. 2012, MNRAS, 421, 2342 
Stetson, P. B. 1987, PASP, 99, 191

Swift, J. J., Johnson, J. A., Morton, T. D., et al. 2013, ApJ, 764, 105

Torres, G. 2013, Astronomische Nachrichten, 334, 4

Torres, G., Fressin, F., Batalha, N. M., et al. 2011, ApJ, 727, 24

Torres, G., Konacki, M., Sasselov, D. D., \& Jha, S. 2004, ApJ, 614, 979

Torres, G., Winn, J. N., \& Holman, M. J. 2008, ApJ, 677, 1324

Tsuji, T., Ohnaka, K., \& Aoki, W. 1996, A\&A, 305, L1
Twicken, J. D., Clarke, B. D., Bryson, S. T., et al. 2010, Proc. SPIE, 7740, 774023

van Belle, G. T., \& von Braun, K. 2009, ApJ, 694, 1085

Weiss, L. M., Marcy, G. W., Rowe, J. F., et al. 2013, ApJ, 768, 14

Winn, J. N., Holman, M. J., Torres, G., et al. 2008, ApJ, 683, 1076

Winn, J. N., Matthews, J. M., Dawson, R. I., et al. 2011, ApJL, 737, L18

Wright, N. J., Drake, J. J., Mamajek, E. E., \& Henry, G. W. 2011, ApJ, 743, 48 